\title{
A systematic review of clinical and surgical predictors of complications following surgery for degenerative cervical myelopathy
}

\author{
Lindsay Tetreault, BSc, ${ }^{1}$ Ahmed Ibrahim, MD, FRCS, PhD, ${ }^{1}$ Pierre Côté DC, PhD, ${ }^{2}$ \\ Anoushka Singh, PhD, ${ }^{1}$ and Michael G. Fehlings, MD, PhD, FRCSC ${ }^{1}$
}

${ }^{1}$ Toronto Western Hospital, University Health Network; and 2University of Ontario Institute of Technology (UOIT), UOIT-CMCC Centre for the Study of Disability Prevention and Rehabilitation, Toronto, Ontario, Canada

OBJECTIVE Although generally safe and effective, surgery for the treatment of cervical spondylotic myelopathy (CSM) is associated with complications in $11 \%-38 \%$ of patients. Several predictors of postoperative complications have been proposed but few are used to detect high-risk patients. A standard approach to identifying "at-risk" patients would improve surgeons' ability to prevent and manage these complications. The authors aimed to compare the complication rates between various surgical procedures used to treat CSM and to identify patient-specific, clinical, imaging, and surgical predictors of complications.

METHODS The authors conducted a systematic review of the literature and searched MEDLINE, MEDLINE in Process, EMBASE, and Cochrane Central Register of Controlled Trials from 1948 to September 2013. Cohort studies designed to evaluate predictors of complications and intervention studies conducted to compare different surgical approaches were included. Each article was critically appraised independently by 2 reviewers, and the evidence was synthesized according to the principles outlined by the Grading of Recommendation Assessment, Development and Evaluation (GRADE) Working Group.

RESULTS A total of 5472 citations were retrieved. Of those, 60 studies met the inclusion criteria and were included in the review. These studies included 36 prognostic cohort studies and 28 comparative intervention studies. High evidence suggests that older patients are at a greater risk of perioperative complications. Based on low evidence, other clinical factors such as body mass index, smoking status, duration of symptoms, and baseline severity score, are not predictive of complications. With respect to surgical factors, low to moderate evidence suggests that estimated blood loss, surgical approach, and number of levels do not affect rates of complications. A longer operative duration (moderate evidence), however, is predictive of perioperative complications and a 2-stage surgery is related to an increased risk of major complications (high evidence). In terms of surgical techniques, higher rates of neck pain were found in patients undergoing laminoplasty compared with anterior spinal fusion (moderate evidence). In addition, with respect to laminoplasty techniques, there was a lower incidence of C-5 palsy in laminoplasty with concurrent foraminotomy compared with nonforaminotomy (low evidence).

CONCLUSIONS The current review suggests that older patients are at a higher risk of perioperative complications. A longer operative duration and a 2-stage surgery both reflect increased case complexity and can indirectly predict perioperative complications.

http://thejns.org/doi/abs/10.3171/2015.3.SPINE14971

KEY WORDS cervical spondylotic myelopathy; systematic review; surgery; complications; degenerative

$\mathrm{C}$ ERVICAL spondylotic myelopathy (CSM) is a progressive degenerative spine disease and the most common cause of spinal cord dysfunction in adults worldwide. ${ }^{33,65}$ Although generally safe and effective, surgery for the treatment of CSM is associated with compli- cations in $11 \%-38 \%$ of patients. ${ }^{6,15}$ These include dysphagia, C-5 radiculopathy, wound infection, axial pain, and postoperative kyphosis.

Guidelines for the surgical management of CSM are rapidly evolving, and surgeons must consider several vari-

ABBREVIATIONS ACDF = anterior cervical discectomy and fusion; $\mathrm{ADF}=$ anterior decompression and fusion; $\mathrm{BMI}=\mathrm{body}$ mass index; $\mathrm{CDH}=\mathrm{cervical}$ disc herniation; CSM = cervical spondylotic myelopathy; EOLP = expansive open-door laminoplasty; GRADE = Grades of Recommendation Assessment, Development and Evaluation; ICD = International Classification of Disease; JOA = Japanese Orthopaedic Association; $K Q=$ key question; OPLL = ossification of the posterior longitudinal ligament; PCB = plate cage Benezech.

SUBMITTED September 25, 2014. ACCEPTED March 26, 2015.

INCLUDE WHEN CITING Published online September 25, 2015; DOI: 10.3171/2015.3.SPINE14971. 
ables when selecting a surgical approach. ${ }^{69}$ These include comorbidities, pathology, clinical presentation, level of stenosis, cervical sagittal alignment, presence of axial neck pain, and familiarity with the technique. ${ }^{46}$ In addition, the safety of the procedure and risks of complications also inform the surgical decision-making process. Historically, posterior laminectomy was considered to be the gold standard and treatment choice for CSM; however, the risk of surgery-related complications including postoperative kyphosis, segmental instability, and progression of myelopathy has promoted the addition of fusion to simple laminectomy. Unfortunately, fusion does affect the biomechanics of the cervical spine and can lead to decreased mobility, neck stiffness, and adjacent segment degeneration. Laminoplasty was later developed to enlarge the spinal canal while preserving neck motion and spinal curvature. However, this technique is contraindicated in patients with neutral or kyphotic alignment and can lead to worsening or persistence of preexisting neck pain. The anterior approach, on the other hand, has recently grown in popularity and is becoming the preferred method of decompression for the majority of CSM cases.

Although the rate of complications associated with surgery are well documented, we lack comparative measures of complication rates between surgical approaches and techniques. This knowledge gap is important as this information can help surgeons balance the risks and benefits of each procedure. Furthermore, several variables may be predictive of postoperative complications, including specific patient characteristics, imaging parameters, and other surgical factors. By identifying these key predictors, clinicians will be better equipped to detect their high-risk patients and take appropriate preoperative and intraoperative preventative measures to optimize patient outcome.

This systematic review addresses 2 key questions (KQs). KQ1: Are there clinical, imaging, or surgical factors associated with complications during or following surgery? KQ2: Do the rates of complications differ across surgical interventions?

\section{Methods \\ Eligibility Criteria \\ Population}

Our review targeted studies of adult patients $(>18$ years) with cervical myelopathy secondary to spondylosis, disc herniation, ossification of the posterior longitudinal ligament (OPLL), congenital stenosis, or subluxation. All patients were treated surgically and were followed up postoperatively. Studies were excluded if they included patients with traumatic spinal cord injury, thoracic or lumbar myelopathy, radiculopathy, tumor, or infection. Furthermore, studies were excluded if patients were undergoing revision surgery (Table 1).

\section{Prognostic Factors}

For KQ1, we sought studies that evaluated various predictors of complications including clinical, imaging, and surgical factors. Studies were excluded if their focus was on the predictive value of neuromonitoring or surgical materials. For KQ2, we focused on studies that compared complication rates between different surgical approaches or techniques (Table 1).

\section{Outcomes}

Our primary outcome measure was overall postoperative complication rates or rates of specific complications such as C-5 nerve root palsy, dysphagia, infection, or instability. Studies that focused on predictors of heterotopic ossification, progression of myelopathy, or adjacent segment degeneration were excluded. Studies that defined axial pain and postoperative kyphosis as complications were included. However, those that discussed axial pain and sagittal alignment as measures of pain and radiographic outcomes, respectively, were excluded (Table 1).

\section{Study Characteristics}

For KQ1, we sought cohort studies designed to evaluate the association between complications and clinical, imaging, and surgical factors using a multivariate analysis. For the sake of thoroughness, studies that did not conduct a multivariate analysis were still summarized but were not included when rating the overall body of evidence. For KQ2, we focused on cohort or case-control studies that measured the association between surgical approach or technique and complications, while controlling for important confounders. For both KQ1 and KQ2, studies had to report $\mathrm{p}$ values to be included.

\section{Information Sources}

Studies were identified using 4 electronic databases: MEDLINE, MEDLINE in Process, EMBASE, and Cochrane Central Register of Controlled Trials. The last search was performed on September 23, 2013.

\section{Search}

We developed a search strategy with a librarian who specializes in neuroscience research. The strategy was first developed in MEDLINE and then appropriately modified for the other 3 databases. We used the following terms to search all databases: CSM or OPLL AND Prediction or Risk of Complications arising from Surgery or Neurosurgery. Only studies involving humans and written in English were considered for inclusion, with no other limits applied.

\section{Study Selection}

All abstracts and titles were reviewed independently in an unblinded, standardized manner by 2 of the authors (L.T., A.S.). The abstracts were sorted by our predefined inclusion criteria and were classified as relevant, possibly relevant, or irrelevant. Full text investigation of the possibly relevant studies was done for further clarification. Disagreement between reviewers was resolved through discussion.

\section{Data Extraction and Synthesis}

The following data were extracted from each included article: study design; patient sample and characteristics, including diagnosis and treatment administered; clinical, 
TABLE 1. Inclusion and exclusion criteria for studies reviewed

\begin{tabular}{|c|c|c|}
\hline Characteristic & Inclusion & Exclusion \\
\hline Patients & $\begin{array}{l}\text { Patients w/ cervical myelopathy secondary to spondylosis, disk herniation, OPLL, } \\
\text { congenital stenosis, \& subluxation (DCM); treated surgically; age }>18 \mathrm{yrs}\end{array}$ & $\begin{array}{l}\text { Patients w/ traumatic spinal cord injury, thoracic } \\
\text { or lumbar myelopathy, tumor, infection radicu- } \\
\text { lopathy or other nondegenerative myelopathy; } \\
\text { patients undergoing revision surgery }\end{array}$ \\
\hline $\begin{array}{l}\text { Prognostic } \\
\text { factors }\end{array}$ & $\begin{array}{l}\text { Clinical factors: age, duration of symptoms, baseline severity score, signs, symp- } \\
\text { toms, comorbidities, smoking status, sex, BMI, diagnosis } \\
\text { Imaging factors: signal changes on T1- or T2-WI, transverse area, AP diameter, } \\
\text { no. of compressed segments, level of max compression, compression ratio, } \\
\text { shape of OPLL lesion, cervical alignment, range of motion } \\
\text { Surgical factors: surgical approach, technique, no. of operated segments, no. of } \\
\text { stages, fusion, operative time, EBL }\end{array}$ & $\begin{array}{l}\text { Different types of surgical materials (e.g., drills, } \\
\text { grafting material), neuromonitoring }\end{array}$ \\
\hline Outcome & $\begin{array}{l}\text { Postop complications: nerve root palsy, axial pain, surgical, dysphagia, infection, } \\
\text { instability, systemic }\end{array}$ & $\begin{array}{l}\text { Heterotopic ossification, progression of myelopa- } \\
\text { thy, ASD }\end{array}$ \\
\hline Study design & $\begin{array}{l}\text { KQ1: cohort studies designed to evaluate prognostic factors using multivariate } \\
\text { analyses } \\
\text { KQ2: cohort or case-controlled studies designed to compare complication rates } \\
\text { between } 2 \text { surgical groups, while controlling for important confounders } \\
\text { Must report p values or ORs }\end{array}$ & $\begin{array}{l}\text { Review articles, opinions, studies } w /<15 \text { pa- } \\
\text { tients, animal or biomechanical studies }\end{array}$ \\
\hline
\end{tabular}

$\mathrm{AP}=$ anteroposterior; $\mathrm{ASD}$ = adjacent-segment degeneration; $\mathrm{DCM}$ = degenerative cervical myelopathy; $\mathrm{EBL}=$ estimated blood loss; $\mathrm{OR}=$ odds ratio; $\mathrm{T} 1-\mathrm{WI}=\mathrm{T} 1-$ weighted MRI; T2-WI = T2-weighted MRI.

imaging, and surgical prognostic factors evaluated; primary complication outcome; and results of association, including reported odds ratios, regression coefficients, or $\mathrm{p}$ values.

\section{Risk of Bias in Individual Studies}

The class of evidence for each article was rated (Class I, II, III, IV) independently by 2 reviewers using criteria outlined by the Journal of Bone and Joint Surgery for prognostic (KQ1) and therapeutic (KQ2) studies and modified to encompass both methodological quality and risk of bias. Prognostic studies included in this review were all cohort studies and were assessed based on whether patients were at a similar time point in their disease/treatment, the rate of follow-up, and whether the analysis controlled for important confounders. Therapeutic studies were rated based on study design, patient selection, if the analysis included intention to treat, blinded assessment, whether cointerventions were equally applied, rates of follow-up, statistical power, and control for confounding. The level of evidence for therapeutic studies in this review was judged based on how the study was designed to compare and analyze rates of complications across surgical cohorts.

\section{Risk of Bias Across Studies}

The overall body of evidence was then assessed using a scoring system developed by the Grading of Recommendation Assessment, Development and Evaluation (GRADE) working group with recommendations from the Agency for Healthcare Research and Quality (AHQR). ${ }^{1}$ For KQ1, we only considered results from studies conducting a multivariate analysis.

The initial strength of the overall body of evidence was graded and classified as "high" if the majority of studies were Class I or II and "low" if the majority of the studies were Class III or IV. The body of evidence was downgraded 1 or 2 levels if the results were inconsistent, the evidence was indirect, the effect estimates were imprecise, or if there was a non a priori statement of subgroup analyses. In contrast, the body of evidence was upgraded 1 or 2 levels if there was a large magnitude of effect or a dose-response gradient. The final rating of the body of evidence expresses our confidence in the estimate of effect and the impact of further research on this topic. An overall strength of high means we have high confidence that the evidence reflects the true effect. Further research is very unlikely to change our confidence in the estimate of effect. The overall strength of "moderate" means we have moderate confidence that the evidence reflects the true effect. Further research may change our confidence in the estimate of effect and may change the estimate. A grade of low means we have low confidence that the evidence reflects the true effect. Further research is likely to change the confidence in the estimate of effect and likely to change the estimate. Finally, a grade of "insufficient" means that evidence either is unavailable or does not permit a conclusion.

\section{Results \\ Study Selection}

The search yielded a total of 5472 citations. After initial review of abstracts and titles, 5259 studies did not meet our inclusion criteria. Following full text investigation, an additional 153 studies were excluded because 1) not all patients were diagnosed with degenerative cervical myelopathy, 2) p values were not provided for analyses, and/or 3) a statistical test was not used to compare complication rates or demographic differences between 2 groups. 
A total of 60 studies were deemed relevant following this rigorous review process.

\section{Study Characteristics}

For KQ1, we identified 36 prognostic cohort studies discussing key clinical, imaging, or surgical predictors of complications. Of these studies, only 9 (3 prospective, 6 retrospective) conducted a multivariate analysis and controlled for potential confounding variables. ${ }^{3,13,19,21,26,34,36,37,41}$ Sample sizes ranged from 81 to 58,118 surgical patients, with mean ages between 57 and 64.1 years. All patients were diagnosed with some form of degenerative cervical myelopathy, with the majority presenting with either CSM or OPLL. The main outcome was postoperative complications in 4 studies, ${ }^{3,13,19,21}$ C-5 or upper extremity palsy in $3,{ }^{26,34,41}$ major intraoperative blood loss in $1,{ }^{37}$ and axial pain in $1 .{ }^{36}$ As indicated by Table 2, several clinical, imaging, and surgical predictors were evaluated in these studies. Twenty-seven additional papers reported on predictors of complications but did not use an adequate multivariate statistical analysis. The following is a summary of the primary outcomes of these studies: postoperative complications $(\mathrm{n}=4) ; ;^{20,27,50,52}$ nerve root palsy $\left.(\mathrm{n}=8)\right)^{7-9,12,31,42,49,53}$ radiculopathy $(\mathrm{n}=3) ; 24,40,67$ shoulder stiffness and neck pain $(\mathrm{n}=1) ;{ }^{76}$ instability $(\mathrm{n}=1) ;{ }^{25}$ postoperative kyphosis $(\mathrm{n}=2) ;{ }^{34,64}$ axial pain $(\mathrm{n}=3) ; 54,55,61$ pseudarthrosis $(\mathrm{n}=1) ;{ }^{16}$ graft dislodgement $(n=2) ; ;^{16,45}$ closure of lamina $(n=2) ; 1,68$ and reconstruction failure $(\mathrm{n}=1) .{ }^{56}$

For KQ2, a total of 28 therapeutic cohort studies (2 randomized control trials, 11 prospective, 15 retrospective) met our inclusion criteria. These studies were designed to compare the efficacy and complication rates between 2 different surgical interventions or varying techniques. Studies reported on differences between anterior and posterior approaches $(\mathrm{n}=9) ; 15,18,19,22,30,42,44,48,59$ laminoplasty and laminectomy and fusion $(\mathrm{n}=4) ; 10,19,70,72$ anterior decompression and Bryan disc $(\mathrm{n}=1) ;{ }^{11}$ and anterior cervical discectomy and fusion (ACDF) and corpectomy (n $=2){ }^{47,63}$ In addition, 11 studies compared laminoplasty techniques or considered technical differences such as laminoplasty with and without foraminotomy or muscle preservation. . $^{28,29,34,38,39,43,57,58,60,66,71}$

\section{Risk of Bias}

For the prognostic studies, we critically appraised 9 studies in which a multivariate analysis was conducted. Of these, 3 were considered Class II and 6 were rated Class III. The 3 Class II studies were prospective in nature, had adequate follow-up rates, and accounted for key confounding variables in their analyses. The Class III studies had moderately high risk of bias as most were retrospective cohort studies that violated $\geq 1$ criteria required for a good-quality (Class II) cohort study.

For the therapeutic studies, the majority were graded Class III as they were moderate or poor-quality cohort studies. These studies were downgraded from Class II to Class III because cointerventions were not applied equally; assessment was not blinded in prospective studies; data were unreliable in retrospective studies; followup rates were $<80 \%$; and/or analyses did not control for important confounders. Three studies were Class II evidence.

\section{Results of Individual Studies \\ KQ1: Are There Clinical or Imaging Factors That Can Predict Perioperative Complications? \\ Complications}

Three studies considered age and comorbidities as important clinical predictors of perioperative complications. ${ }^{3,19,21}$ Boakye et al. reported that patients aged 65 years or older were 2.28 (OR 2.28, 95\% CI 1.74-2.98) times more likely to experience complications following spinal fusion than those aged 18 to 44 years. Furthermore, patients 85 years or older were at an even higher risk of postoperative complications (OR 5.1, 95\% CI 3.08-8.35). In a study by Fehlings et al., age was considered to be an important predictor of minor and major perioperative complications (OR 1.029, 95\% CI 1.002-1.057, $\mathrm{p}=0.035$ ). Similarly, Furlan et al. concluded that age was significantly associated with postoperative complications (OR 1.09, 95\% CI 1.015-1.172, $\mathrm{p}=0.018$ ).

According to Boakye et al., patients with 3 or more comorbidities were twice (OR 1.98, 95\% CI 1.59-2.48) as likely to experience complications following spinal fusion than healthy individuals. In an analysis by Fehlings et al., a comorbidity score was developed to encompass both number of comorbidities and severity of disease. Patients who experienced major or minor perioperative complications did not have a significantly different comorbidity score than those who did not $(\mathrm{p}=0.84)$. In a univariate analysis by Furlan et al., there was a greater number of comorbidities, as defined by the number of International Classification of Disease (ICD)-9 codes, in the complication group compared with the noncomplication group $(\mathrm{p}=0.033)$. In addition, the mean Charlson Comorbidity Index was higher in patients with postoperative complications, although this difference did not reach statistical significance $(\mathrm{p}=0.092)$. In multivariate analysis, however, neither the number nor the severity of comorbidities were found to be predictive.

Cook et al. focused on the impact of diabetes on various complication categories, including dysphagia, postoperative infection, and procedural. ${ }^{13}$ In univariate analysis, patients with diabetes experienced a higher rate of dysphagia $(\mathrm{p}<0.01)$, transfusion $(\mathrm{p}=0.01)$ and cardiac $(\mathrm{p}$ $<0.01)$, peripheral vascular $(\mathrm{p}=0.01)$, respiratory $(\mathrm{p}<$ $0.01)$, and hematoma complications $(\mathrm{p}=0.01)$ than $\mathrm{pa}-$ tients without diabetes. In addition, cardiac $(\mathrm{p}=0.01)$ and hematoma $(p=0.01)$ complications were more prevalent in patients with uncontrolled diabetes as compared to those with controlled disease. There were no significant differences in complication rates between patients with Type I and Type II diabetes. Following adjustment for confounders, patients with diabetes were at a higher risk of cardiac complications than those without diabetes (OR $1.57,95 \%$ CI 1.14-2.16, $\mathrm{p}=0.01$ ). In addition, patients with uncontrolled diabetes were more likely to experience cardiac complications (OR 2.82, 95\% CI 1.14-7.01, $\mathrm{p}=0.03$ ), complications of hematomas (OR 5.13, 95\% CI 2.16-12.17, $\mathrm{p}<0.01$ ), and postoperative infection (OR 
TABLE 2. Demographics and characteristics of complication prognostic studies with multivariate analysis

\begin{tabular}{|c|c|c|c|c|c|c|c|}
\hline $\begin{array}{l}\text { Authors \& Year; } \\
\text { Design (class) }\end{array}$ & Sex, $\%$ & $\begin{array}{l}\text { Mean Age } \\
\text { (range) }^{*}\end{array}$ & Characteristic, $n$ & Surgery & $\begin{array}{l}\text { Clinical or Imaging } \\
\text { Factors Assessed }\end{array}$ & $\begin{array}{l}\text { Surgical Factors } \\
\text { Assessed }\end{array}$ & Complications \\
\hline $\begin{array}{l}\text { Boakye et al., } \\
\text { 2008; RC } \\
\text { (III) }\end{array}$ & $M, 58.1 \%$ & NA & $\operatorname{CSM}(n=58,115)$ & Spinal fusion & $\begin{array}{l}\text { Age, sex, race, } \\
\text { comorbidities }\end{array}$ & None & $\begin{array}{l}\text { Neurological; pul- } \\
\text { monary; thrombo- } \\
\text { embolic, cardiac, } \\
\text { renal, \& hematoma } \\
\text { complications; infec- } \\
\text { tion; hoarseness; } \\
\text { dysphagia }\end{array}$ \\
\hline $\begin{array}{l}\text { Cook et al., } \\
\text { 2008; RC } \\
\text { (III) }\end{array}$ & $M, 56.0 \%$ & NA & $\begin{array}{l}\text { CSM }(n=37,732) \\
\quad \text { diabetes }(n= \\
3,432)\end{array}$ & Spinal fusion & $\begin{array}{l}\text { Diabetes (pres- } \\
\text { ence vs } \\
\text { absence, Type } \\
\text { I vs Type II, } \\
\text { controlled vs } \\
\text { uncontrolled) }\end{array}$ & None & $\begin{array}{l}\text { CNS, respiratory, car- } \\
\text { diac, peripheral vas- } \\
\text { cular, procedural, } \\
\text { wound \& hematoma } \\
\text { complications, CSF } \\
\text { leak or persistent } \\
\text { fistula, carotid or } \\
\text { vertebral artery } \\
\text { injury, transfusion, } \\
\text { postop infection, } \\
\text { hoarseness, dys- } \\
\text { phagia }\end{array}$ \\
\hline $\begin{array}{l}\text { Fehlings et al., } \\
\text { 2012; PC } \\
\text { (II) }\end{array}$ & M, $58.9 \%$ & $57(29-86)$ & $\operatorname{CSM}(n=302)$ & $\begin{array}{l}\text { Anterior }(n=176) \text {, } \\
\text { posterior }(n= \\
107), \text { combined } \\
(n=19)\end{array}$ & $\begin{array}{l}\text { Age, sex, comor- } \\
\text { bidity score, } \\
\text { BMI, baseline } \\
\text { severity, smok- } \\
\text { ing status }\end{array}$ & $\begin{array}{l}\text { Approach, no. of } \\
\text { stages, no. of } \\
\text { levels, spinal } \\
\text { fusion, fusion } \\
\text { approach, } \\
\text { laminectomy, } \\
\text { corpectomy, } \\
\text { operative dura- } \\
\text { tion, blood loss }\end{array}$ & $\begin{array}{l}\text { Minor \& major periop } \\
\text { complications, major } \\
\text { periop complications }\end{array}$ \\
\hline $\begin{array}{l}\text { Furlan et al., } \\
\text { 2011; PC } \\
\text { (II) }\end{array}$ & $M, 70.4 \%$ & $57(32-88)$ & $\operatorname{CSM}(n=81)$ & $\begin{array}{l}\text { Anterior }(n=56) \text {, } \\
\text { posterior }(n= \\
23), \text { combined } \\
(n=2)\end{array}$ & $\begin{array}{l}\text { Age, sex, duration } \\
\text { of symptoms, } \\
\text { comorbidities } \\
\text { (no. \& CCl) }\end{array}$ & $\begin{array}{l}\text { Approach, no. of } \\
\text { levels }\end{array}$ & Postop complications \\
\hline $\begin{array}{l}\text { Hasegawa et } \\
\text { al., 2007; } \\
\text { RC (III) }\end{array}$ & NA & NA & $\begin{array}{l}\operatorname{CSM}(n=587) \\
\operatorname{OPLL}(n=143), \\
\operatorname{CDH}(n=117) \\
\operatorname{OLF}(n=10)\end{array}$ & $\begin{array}{l}\text { ADF }(n=424), \\
\quad \text { laminoplasty } \\
(n=345), \\
\text { laminectomy } \\
(n=88)\end{array}$ & $\begin{array}{l}\text { Age, sex, baseline } \\
\text { severity, diag- } \\
\text { nosis }\end{array}$ & $\begin{array}{l}\text { Procedure, no. of } \\
\text { levels }\end{array}$ & Postop UE palsy \\
\hline $\begin{array}{l}\text { Kaneyama et } \\
\text { al., 2010; } \\
\text { PC (II) }\end{array}$ & $\mathrm{M}, 74.0 \%$ & 64.1 (NA) & $\begin{array}{l}\operatorname{CSM}(n=108) \\
\operatorname{OPLL}(n=31) \\
\operatorname{CDH}(n=7)\end{array}$ & $\begin{array}{l}\text { Open-door lami- } \\
\text { noplasty }(n= \\
73) \text {, double- } \\
\text { door lamino- } \\
\text { plasty }(n=73)\end{array}$ & $\begin{array}{l}\text { Age, sex, baseline } \\
\text { severity, } \\
\text { diagnosis, } \\
\text { physical signst, } \\
\text { radiologic } \\
\text { examsł, CMAP } \\
\text { amplitude }\end{array}$ & $\begin{array}{l}\text { Surgical tech- } \\
\text { nique, no. of } \\
\text { levels }\end{array}$ & C-5 palsy§ \\
\hline $\begin{array}{l}\text { Kato et al., } \\
\text { 2012; RC } \\
\text { (III) }\end{array}$ & $\mathrm{M}, 78.7 \%$ & 62.7 (NA) & OPLL $(n=545)$ & $\begin{array}{l}\text { Open-door } \\
\text { laminoplasty } \\
(n=234), \\
\text { double-door } \\
\text { laminoplasty } \\
\text { ( } n=286) \text {, other } \\
\text { types of lamino- } \\
\text { plasty ( } n=25)\end{array}$ & $\begin{array}{l}\text { Age, sex, comor- } \\
\text { bidities, size of } \\
\text { OPLL, OPLL } \\
\text { type, occupy- } \\
\text { ing ratio, C2-7 } \\
\text { angle, high SI } \\
\text { on T2-WI }\end{array}$ & $\begin{array}{l}\text { Surgical tech- } \\
\text { nique, no. of } \\
\text { levels }\end{array}$ & $\begin{array}{l}\text { Major intraop blood } \\
\text { loss }(>500 \mathrm{~g})\end{array}$ \\
\hline
\end{tabular}


TABLE 2. Demographics and characteristics of complication prognostic studies with multivariate analysis (continued)

\begin{tabular}{|c|c|c|c|c|c|c|c|}
\hline $\begin{array}{l}\text { Authors \& Year; } \\
\text { Design (class) }\end{array}$ & Sex, $\%$ & $\begin{array}{l}\text { Mean Age } \\
\text { (range })^{*}\end{array}$ & Characteristic, $n$ & Surgery & $\begin{array}{l}\text { Clinical or Imaging } \\
\text { Factors Assessed }\end{array}$ & $\begin{array}{l}\text { Surgical Factors } \\
\text { Assessed }\end{array}$ & Complications \\
\hline $\begin{array}{l}\text { Kato et al., } \\
\text { 2008; RC } \\
\text { (III) }\end{array}$ & $M, 69.6 \%$ & $61(38-82)$ & $\begin{array}{l}\text { CSM }(n=135) \\
\quad \text { OPLL }(n=10)\end{array}$ & $\begin{array}{l}\text { Open-door lami- } \\
\text { noplasty }\end{array}$ & $\begin{array}{l}\text { Age, sex, diag- } \\
\text { nosis, duration } \\
\text { of symptoms, } \\
\text { baseline sever- } \\
\text { ity, preop align- } \\
\text { ment, preop } \\
\text { ROM }\end{array}$ & $\begin{array}{l}\text { Operative dura- } \\
\text { tion, blood loss, } \\
\text { preservation } \\
\text { of paraspinal } \\
\text { muscles }\end{array}$ & Axial painף \\
\hline $\begin{array}{l}\text { Kimura et al., } \\
\text { 2012; RC } \\
\text { (III) }\end{array}$ & $M, 75.3 \%$ & $60(31-84)$ & OPLL $(n=150)$ & ADF & $\begin{array}{l}\text { Occupying ratio, } \\
\text { C2-7 angle }\end{array}$ & $\begin{array}{l}\text { Operative dura- } \\
\text { tion, blood loss, } \\
\text { no. of fused } \\
\text { segments }\end{array}$ & Postop UE paresis** \\
\hline
\end{tabular}

$\mathrm{CCl}=$ Charlson Comorbidity Index; $\mathrm{CDH}=$ cervical disc herniation; $\mathrm{CMAP}=$ compound muscle action potential; NA = not available; OLF = ossification of the ligamen-

tum flavum; $\mathrm{PC}=$ prospective cohort; $\mathrm{RC}=$ retrospective cohort; $\mathrm{ROM}=$ range of motion; $\mathrm{SI}$ = signal intensity; UE = upper-extremity.

* Age in years.

$\dagger$ Included muscle strength of deltoid and biceps, muscle stretch reflex of deltoid and biceps tendon, and neurologic impediment level of myelopathy.

$\ddagger$ Included cervical alignment, number of compressed segments, position of the superior articular process, cord inclination, and high-intensity area on T2-weighted image.

$\S$ Deterioration of bicep and deltoid muscle strength by at least 1 level in a standard muscle manual test without any deterioration of other neurological symptoms.

I New development or progression of axial pain at 6 months to 2 years after surgery.

** Deterioration of upper-extremity function.

$7.46,95 \%$ CI $1.33-41.79, \mathrm{p}=0.02$ ) as compared to patients with controlled diabetes.

Other clinical predictors such as sex, duration of symptoms, baseline severity score, smoking status, or body mass index (BMI) were not found to be significantly associated with postoperative complications in either univariate or multivariate analysis (Table 3).,19,21

\section{Upper Extremity or C-5 Palsy}

Hasegawa et al., Kaneyama et al., and Kimura et al. explored various clinical and imaging risk factors of upper extremity palsy following surgical decompression. ${ }^{26,34,41}$ The sample used in the studies by Hasegawa et al. and Kaneyama et al. consisted of surgical patients with various forms of degenerative cervical myelopathy, including CSM, OPLL, and cervical disc herniation (CDH). Both studies found that patients with OPLL as the primary diagnosis were at a markedly higher risk of experiencing upper extremity palsy than those with other forms of degenerative myelopathy (Hasegawa et al., OR 19.0, p < 0.0001; Kaneyama et al., OR 43.8, p < 0.05). In the study by Hasegawa et al., although older age was found to be a significant predictor of upper extremity palsy in univariate analysis (OR 0.1, $\mathrm{p}<0.05$ ), it was not significant in multivariate analysis (OR $2.59, p=0.108$ ). Age was also found to be an insignificant predictor of C-5 palsy in the study by Kaneyama et al.

Kimura et al. conducted a study on 150 OPLL patients to determine the risk factors for neurological complications occurring within 2 weeks of anterior decompression and fusion (ADF). High occupying ratio was the only significant clinical or imaging predictor of postoperative upper extremity paresis (OR 1.047, p = 0.040). Age was not considered in this analysis.

Sex, baseline severity score, and various clinical signs were not significant clinical predictors of upper extremity palsy. ${ }^{26,34}$ Several imaging factors were also not related to postoperative palsy development, including cervical alignment, number of compressed segments, position of superior articular process, cord inclination, signal change on T2-weighted MRI, and mid-C-2 to mid-C-7 angle. ${ }^{34,41}$

\section{Major Blood Loss}

A single study by Kato et al. examined important clinical and imaging predictors of major intraoperative blood loss during laminoplasty in patients with OPLL. ${ }^{37}$ When comparing a group of patients with $>500 \mathrm{~mL}$ blood loss to a group with $\leq 500 \mathrm{ml}$, there were no significant differences with respect to age, sex, size, or type of OPLL (continuous, segmental, mixed, or local), C2-7 alignment, or high signal intensity on T2-weighted MRI. The occupying ratio, however, was significantly higher $(48.3 \% \pm 13.4 \%)$ in the major blood loss group as compared to the control group $(42.7 \% \pm 13.2 \%, p=0.02)$. Furthermore, an occupying ratio of $60 \%$ or greater was the only significant risk factor following stepwise multivariate, logistic regression (OR 2.94, 95\% CI 1.1-5.3, $\mathrm{p}=0.03$ ).

\section{Axial Pain}

Kato et al. defined postoperative axial pain as pain from the nuchal to scapular region developing or progressing between 6 months and 2 years postoperatively. ${ }^{36}$ Following multivariate analysis, older age was the only significant predictor of axial pain (> 63, OR $0.17,95 \%$ CI $0.04-0.72$, $\mathrm{p}>0.05$ ). Sex, diagnosis, duration of symptoms, baseline severity score, preoperative alignment, and range of motion were not significant risk factors.

\section{KQ1: Are There Surgical Factors That Can Predict Complication Development? \\ Complications}

Two studies assessed the value of various surgical factors as predictors of complications. ${ }^{19,21}$ Based on univari- 
TABLE 3. Important clinical, imaging, and surgical predictors of perioperative complications, including nerve root palsy, axial pain, and major intraoperative blood loss*

\begin{tabular}{|c|c|c|c|c|}
\hline $\begin{array}{l}\text { Authors \& } \\
\quad \text { Year }\end{array}$ & $\begin{array}{l}\text { Level of } \\
\text { Evidence }\end{array}$ & Parameters for Univariate Analysis & $\begin{array}{l}\text { Multivariate Analysis } \\
\text { (clinical/imaging factors) }\end{array}$ & $\begin{array}{l}\text { Multivariate Analysis } \\
\text { (surgical factors) }\end{array}$ \\
\hline $\begin{array}{l}\text { Boakye et } \\
\text { al., } 2008\end{array}$ & $\begin{array}{l}\text { Pres/ab of } \\
\text { complica- } \\
\text { tions }\end{array}$ & $\begin{array}{l}\text { Age: } p<0.0001 \text {; comorbidities: } \\
\quad p<0.0001 \text {; sex: } p=0.60 \text {; race: } p= \\
0.48\end{array}$ & 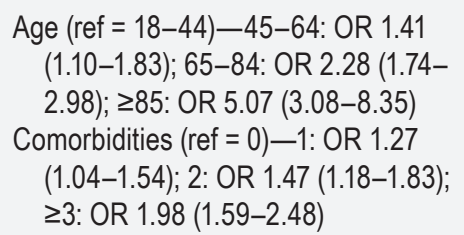 & None \\
\hline $\begin{array}{l}\text { Fehlings et } \\
\text { al., } 2012\end{array}$ & $\begin{array}{l}\text { Pres/ab of } \\
\text { minor \&/ } \\
\text { or major } \\
\text { complica- } \\
\text { tions }\end{array}$ & $\begin{array}{l}\text { Any periop complication-age: } p= \\
\text { 0.006; no. of stages: } p=0.016 ; \\
\text { operative duration: } p=0.009 ; \\
\text { operative blood loss: } p=0.005 ; \text { co- } \\
\text { morbidity score§: } p=0.84 ; \text { sex: } p= \\
\text { 0.75; BMI: } p=0.48 \text {, baseline mJOA: } \\
\text { p=0.28; smoking status: } p=0.47 ; \\
\text { surgical approach: } p=0.11 ; \text { no. of } \\
\text { levels: } p=0.067 ; \text { spinal fusion: } p= \\
\text { 0.82; fusion approach: } p=0.064 ; \\
\text { laminoplasty performed: } p=0.48 ; \\
\text { corpectomy performed: } p=0.84\end{array}$ & $\begin{array}{l}\text { Any periop complication-age, OR } \\
1.029(1.002-1.057), p=0.035 \\
\text { Major complication-age: OR: } 1.054 \\
\quad(1.015-1.094), p=0.006 \text {. } \\
\text { Not significant in either model-sex: } \\
\quad p>0.05 ; \text { comorbidity score§: } \\
\text { p >0.05; BMl: } p>0.05 \text {; smoking } \\
\text { status: } p>0.05 \text {; baseline severity: } \\
p>0.05\end{array}$ & $\begin{array}{l}\text { Any periop complication-operative } \\
\text { duration: OR 1.005 (1.002-1.008), } \\
p=0.001 \text {; no. of stages: } p>0.05 \\
\text { Major complication-combined } \\
\text { anterior-posterior procedure: OR: } \\
5.297 \text { (1.626-17.256), } p=0.006 \text {; } \\
\text { operative duration: } p>0.05 \\
\text { Not significant in either model- } \\
\text { operative blood loss: } p>0.05 \\
\text { approach: } p>0.05 ; \text { no. of levels: } \\
p>0.05 ; \text { laminoplasty: } p>0.05 \text {; } \\
\text { corpectomy: } p>0.05\end{array}$ \\
\hline $\begin{array}{l}\text { Furlan et al., } \\
2011\end{array}$ & $\begin{array}{l}\text { Pres/ab of } \\
\text { complica- } \\
\text { tions }\end{array}$ & $\begin{array}{l}\text { Age: } p=0.006 ; \text { no. of ICD }-9 \text { codes: } \\
\quad p=0.033 ; \text { approach: } p=0.018 ; \text { sex: } \\
p=0.534 ; \text { duration of symptoms: } \\
p=0.106 ; \mathrm{CCl}: p=0.09 ; \text { no. of } \\
\text { levels: } p=0.233\end{array}$ & $\begin{array}{l}\text { Age: OR } 1.09(1.015-1.172), p= \\
\text { 0.018; sex: } p=0.188 ; \text { no. of ICD-9 } \\
\text { codes: } p=0.113 ; \text { duration of symp- } \\
\text { toms: } p=0.309\end{array}$ & $\begin{array}{l}\text { Surgical approach: } p=0.248 ; \text { no. of } \\
\quad \text { levels: } p=0.454\end{array}$ \\
\hline $\begin{array}{l}\text { Cook et al., } \\
2008\end{array}$ & $\begin{array}{l}\text { Pres/ab of } \\
\text { complica- } \\
\text { tion sub- } \\
\text { groups } \neq\end{array}$ & $\begin{array}{l}\text { Diabetes (vs no diabetes) —respira- } \\
\text { tory complications: } p<0.01 \text {; cardiac } \\
\text { complications: } p<0.01 \text {; peripheral } \\
\text { vascular complications: } p=0.01 \text {; } \\
\text { complications of hematomas: } p= \\
0.01 \text {; transfusion: } p=0.01 \text {; dyspha- } \\
\text { gia: } p<0.01 \text {; other complications: } \\
p=0.001 \\
\text { Controlled diabetes (vs uncon- } \\
\text { trolled) -cardiac complications: } p= \\
0.01 \text {; complications of hematomas: } \\
p=0.01\end{array}$ & $\begin{array}{l}\text { Diabetes (ref = no diabetes) - cardiac } \\
\text { complications: OR 1.57 (1.14-2.16), } \\
p=0.01 \text {; other complications: OR } \\
1.54 \text { (1.17-2.01), } p=0.01 \\
\text { Uncontrolled diabetes (ref = controlled } \\
\text { diabetes) -cardiac complications: } \\
\text { OR } 2.82 \text { (1.14-7.01), } p=0.03 ; \\
\text { complications of hematomas: OR } \\
5.13 \text { (2.16-12.17), } p<0.01 ; \text { postop } \\
\text { infection: OR 7.46 (1.33-41.79), } \\
p=0.02\end{array}$ & \\
\hline $\begin{array}{l}\text { Hasegawa } \\
\text { et al., } \\
2007\end{array}$ & $\begin{array}{l}\text { Pres/ab of UE } \\
\text { palsy }\end{array}$ & $\begin{array}{l}\text { All patients, anterior procedure-age: } \\
\text { p<0.05, <0.01; diagnosis: <0.0001, } \\
<0.001 \text {; no. of levels: } p=0.0591 \\
<0.001 \text {; procedure: NS, NA; sex: } \\
\text { NS, NS; baseline severity score: } \\
\text { NS, NS }\end{array}$ & $\begin{array}{l}\text { All patients-diagnosis (OPLL): OR } \\
\quad 19.0, p<0.0001 \text {; age: OR } 2.59, p= \\
\quad 0.108 \\
\text { Anterior procedure-age OR: } 3.13 \text {, } \\
\quad p=0.076\end{array}$ & $\begin{array}{l}\text { Anterior procedure-no. of levels: } \\
\text { OR } 11.5, p<0.001\end{array}$ \\
\hline $\begin{array}{l}\text { Kaneyama } \\
\text { et al., } \\
2010\end{array}$ & $\begin{array}{l}\text { Pres/ab of } \\
\text { C-5 palsy }\end{array}$ & Approach: $p<0.05$ & $\begin{array}{l}\text { Diagnosis (OPLL): OR } 43.8(1.03- \\
\text { 999.99), } p=0.048 ; \text { age: } p=0.964 ; \\
\text { sex: } p=0.252 ; \text { baseline severity: } \\
p=0.219 ; \text { physical signs } \rrbracket: p= \\
0.117-0.998 ; \text { radiologic exams }{ }^{* *}: \\
p=0.101-0.314 ; \text { CMAP amplitude: } \\
p=0.112-0.291\end{array}$ & $\begin{array}{l}\text { Open-door laminoplasty (vs double- } \\
\text { door): OR } 69.6 \text { (1.14-999.99), } \\
p=0.043 ; \text { no. of opened laminae: } \\
p=0.493\end{array}$ \\
\hline $\begin{array}{l}\text { Kimura et } \\
\text { al., } 2012\end{array}$ & $\begin{array}{l}\text { Pres/ab of } \\
\text { UE paresis }\end{array}$ & $\begin{array}{l}\text { Occupying ratio: } p=0.005 ; \text { blood loss: } \\
\begin{array}{l}p=0.021 ; \text { operative duration: } p= \\
0.002 ; \text { long fusion ( } \geq 3 \text { segments): } \\
p=0.017 ; C 2-7 \text { angle: } p=0.758\end{array}\end{array}$ & $\begin{array}{l}\text { Occupying ratio: } 1.047 \text { (1.002-1.093), } \\
\qquad p=0.040\end{array}$ & $\begin{array}{l}\text { Blood loss: } 1.002 \text { ( } 1.000-1.003), p= \\
\text { 0.047; operative duration : } p= \\
\text { 0.74; long fusion ( } \geq 3 \text { segments): } \\
p=0.77\end{array}$ \\
\hline
\end{tabular}


TABLE 3. Important clinical, imaging, and surgical predictors of perioperative complications, including nerve root palsy, axial pain, and major intraoperative blood loss* (continued)

\begin{tabular}{|c|c|c|c|c|}
\hline $\begin{array}{l}\text { Authors \& } \\
\text { Year }\end{array}$ & $\begin{array}{l}\text { Level of } \\
\text { Evidence }\end{array}$ & Parameters for Univariate Analysis & $\begin{array}{l}\text { Multivariate Analysis } \\
\text { (clinical/imaging factors) }\end{array}$ & $\begin{array}{l}\text { Multivariate Analysis } \\
\text { (surgical factors) }\end{array}$ \\
\hline $\begin{array}{l}\text { Kato et al., } \\
\quad 2012\end{array}$ & $\begin{array}{l}\text { Pres/ab } \\
\text { of major } \\
\text { intraopera- } \\
\text { tive blood } \\
\text { loss }\end{array}$ & $\begin{array}{l}\text { Occupying ratio: } p=0.02 ; \text { operative } \\
\text { duration: } p<0.001 ; \text { diabetes: } p= \\
\text { 0.30; hypertension: } p=0.1 \text {; no. of } \\
\text { levels: } p=0.68 ; \text { sex: } p=0.17 \text {; age: } \\
\text { p = 0.10; size of OPLL: } p=0.23 \text {; } \\
\text { OPLL type: } p=0.14 ; C 2-7 \text { angle: } \\
p=0.93 ; \text { high SI on T2-WI: } p=0.51\end{array}$ & $\begin{array}{l}\text { Occupying ratio ( } \geq 60 \%) \text { : OR } 2.4 \\
(1.1-5.3), p=0.03 ; \text { age: } p>0.05 \text {; } \\
\text { sex: } p>0.05 \text {; size of OPLL: } \\
p>0.05 ; \text { OPLL type: } p>0.05\end{array}$ & \\
\hline $\begin{array}{l}\text { Kato et al., } \\
2008\end{array}$ & $\begin{array}{l}\text { Pres/ab of } \\
\text { postop } \\
\text { axial pain }\end{array}$ & $\begin{array}{l}\text { No factors were significant in univari- } \\
\text { ate analysis (all factors analyzed } \\
\text { are shown in adjacent columns) }\end{array}$ & $\begin{array}{l}\text { Age (>63 yrs): OR } 0.17(0.04-0.72) \\
\text { p <0.05; sex: } p>0.05 \text {; diagnosis: } \\
\text { p >0.05; duration of symptoms: } \\
p>0.05 \text {; baseline severity: } p>0.05 \text {; } \\
\text { preop alignment: } p>0.05 \text {; preop } \\
\text { ROM: } p>0.05\end{array}$ & $\begin{array}{l}\text { Preserving of paraspinal muscles } \\
\text { attached at C-2 (vs nonpre- } \\
\text { serving): OR } 0.13(0.02-0.98) \\
p<0.05 \text {; operative time: } p>0.05 \text {; } \\
\text { blood loss: } p>0.05 \text {; preserving of } \\
\text { paraspinal muscles attached at } \\
\text { C-7 (vs nonpreserving): } p>0.05\end{array}$ \\
\hline
\end{tabular}

exams = examinations; $\mathrm{mJOA}=$ modified JOA; NA = not applicable; NS = not significant; Pres/ab = presence or absence.

* Results of univariate and multivariate analyses.

$\dagger$ All study designs were multiple logistic regression analysis.

‡ Multiple logistic regression analysis controlling for age, discharge year, race, sex, household income, hospital size, hospital type, primary insurance payer, and number of levels fused.

$\S$ Includes both severity and number of comorbidities (1 for mild, 2 for moderate, 3 for severe) summed over several types of comorbidities.

ף Included muscle strength of deltoid and biceps, muscles stretch reflex of deltoid and biceps tendon, and neurologic impediment level of myelopathy.

** Included cervical alignment, number of compressed segments, position of the superior articular process, cord inclination, and high-intensity area on T2-weighted image.

ate analysis, a 2-stage anteroposterior surgery $(\mathrm{p}=0.016)$, a longer operative duration ( $\mathrm{p}=0.009)$, and greater blood loss $(\mathrm{p}=0.005)$ were significantly associated with perioperative complications. Following multivariate analysis, however, operative time was the only significant surgical predictor of overall (major and minor) perioperative complications (OR $1.005,95 \%$ CI $1.002-1.008, \mathrm{p}=0.001) .{ }^{19}$ Fehlings et al. also explored risk factors for only major complications, defined as an event resulting in permanent or prolonged morbidity, prolongation of hospital stay, or invasive intervention. ${ }^{19}$ Patients who were treated surgically with a 2-stage procedure were 5.30 times more (OR 5.30, 95\% CI 1.626-17.256) likely to experience a major complication as compared to those undergoing either a single-stage anterior or posterior surgery $(p=0.006)$. Furlan et al. reported that the anterior approach was associated with fewer postoperative complications than either a posterior or circumferential procedure $(p=0.018) .{ }^{21}$ This surgical factor, however, was not a significant predictor following multivariate analysis $(\mathrm{p}=0.248)$.

Fehlings et al. identified no differences in surgical approach $(\mathrm{p}=0.11)$ or number of vertebrae operated on $(\mathrm{p}$ $=0.067)$ between patients who experienced perioperative complications and those who did not. Furthermore, whether the procedure included spinal fusion $(\mathrm{p}=0.82)$ (anterior or posterior, $\mathrm{p}=0.064)$, a laminoplasty $(\mathrm{p}=0.48)$, or a corpectomy $(\mathrm{p}=0.84)$ did not influence postoperative complication rates.

\section{Upper Extremity or C-5 Palsy}

Hasegawa et al. reported that surgical approach (anterior vs posterior or $\mathrm{ADF}$ vs laminoplasty vs laminectomy) did not influence the occurrence of C-5 palsy. ${ }^{26}$ The num- ber of decompressed levels tended to be higher in patient with C-5 palsy, although this relationship did not reach statistical significance. Following stepwise logistic regression, a greater number of levels was predictive of postoperative $\mathrm{C}-5$ palsy in patients undergoing $\mathrm{ADF}(\mathrm{OR} 11.5, \mathrm{p}<0.001)$.

In a study by Kimura et al., patients with fusion of 3 or more levels $(\mathrm{p}=0.017)$ and a longer operative duration $(\mathrm{p}=0.002)$ were at an increased risk of developing upperextremity palsy. ${ }^{41}$ These 2 relationships, however, were not significant in multivariate analysis $(\mathrm{p}=0.77, \mathrm{p}=0.74$, respectively). Increased intraoperative blood loss was the only identified surgical predictor of upper-extremity palsy (OR $1.002,95 \%$ CI $1.000-1.003, \mathrm{p}=0.047)$.

Finally, Kaneyama et al. reported that patients undergoing open-door laminoplasty were at a significantly higher risk of developing C-5 nerve palsy than those treated with double-door laminoplasty (OR 69.6, 95\% CI 1.14-999.99, $\mathrm{p}=0.043){ }^{34}$ This estimate, however, is unstable. Number of open laminae was the only other surgical factor considered in this study and was not predictive of C-5 palsy ( $p$ $=0.483$ ).

\section{Major Blood Loss}

There was no difference in the number of operated levels between patients who experienced excessive blood loss $(5.1 \pm 1.0)$ and those who did not $(5.0 \pm 0.8, \mathrm{p}=0.68) .{ }^{37} \mathrm{Op}-$ erative duration, however, was longer in the major blood loss group $(240 \pm 112$ minutes vs $149 \pm 59$ minutes, $\mathrm{p}<$ $0.001)$.

\section{Axial Pain}

Based on multivariate analysis, preservation of paraspi- 
nal muscles attached at C-2 decreased the risk of postoperative axial pain (OR 0.13, 95\% CI 0.02-0.98, p <0.05). ${ }^{36}$ Operative duration, blood loss, and preservation of paraspinal muscles attachment at C-7 were not significantly associated with postoperative axial pain.

\section{Results of Studies Without Multivariate Analyses}

Table 4 summarizes the results of studies that identified important predictors of complications but did not conduct a multivariate analysis.

Based on the results of 2 studies, preoperative severity was not related to overall complication rates. ${ }^{20,27}$ In a study by Holly et al., complication rates were compared between patients older than 75 years and patients under 65 years. In the elderly group, the complication rate $(38 \%)$ was significantly higher than in the younger group $(6 \%, \mathrm{p}$ $=0.002$ ). In a study by Lu et al., the incidence of complications was also much higher $(35 \%)$ in the aged group $(\geq$ 70 years) compared with the control group $(9.7 \%)(<70$ years), although this relationship did not reach statistical significance. ${ }^{50}$

There was consensus in the literature that age $(\mathrm{n}=$ $4)^{7,31,43,53} \operatorname{sex}(n=2),{ }^{7,31}$ preoperative severity $(n=5), 7,12,31,43,53$ muscle manual test results $(n=2),{ }^{7,43}$ duration of symptoms $(\mathrm{n}=2),{ }^{31,43}$ diagnosis $(\mathrm{n}=2),{ }^{31,53}$ preoperative cervical lordosis $(n=2), 31,53$ and cervical curvature $(n=2)^{43,49}$ are not significant predictors of nerve root palsy. In a study by Imagama et al., patients experiencing C-5 palsy had a smaller C-5 intervertebral foramen width and a higher degree of anterior protrusion of the superior articular process of C-5. Komagata et al., however, noted no significant difference in the extent of anterior protrusion between patients with $(4.3 \pm 4)$ and without $(3.73 \pm 3)$ palsy $(\mathrm{p}=0.51) .{ }^{31,42}$ Hyperintensity on a MRI was also not an important imaging predictor of nerve root palsy. ${ }^{7,31}$

Three studies examined important predictive factors of radiculopathy following surgery. ${ }^{24,40,65}$ In a study by Greiner-Perth et al., patients who had symptoms of C-5 and/or C-6 radiculopathy were on average older (62.4 vs 58.6 years, $p<0.01$ ) and had a greater number of operated levels $(2.2$ vs 1.7 levels, $\mathrm{p}<0.001)$ than those who did not. The incidence of postoperative radiculopathy, however, was not different between an elderly patient group ( $\geq 70$ years) and a younger patient group ( $<70$ years) in a study by Kawaguchi et al. $(\mathrm{p}=0.18)$. There was consensus in the literature that preoperative severity $(n=2)$ was not significantly associated with postoperative radiculopathy. ${ }^{24,65}$

Risk factors for stability complications such as instability $(\mathrm{n}=1)^{25}$ and postoperative kyphosis $(\mathrm{n}=2)^{35,64}$ were assessed by 3 studies. Guigui et al. reported that patients who were younger $(\mathrm{p}=0.03)$, had a hypermobile spine $(\mathrm{p}<0.0001)$, had greater preoperative range of motion $(\mathrm{p}$ $<0.0001)$, and received a C-2 laminectomy $(\mathrm{p}=0.0164)$ were more susceptible to spinal destabilization. With respect to postoperative kyphosis, a single study reported that a diagnosis of CSM, smaller preoperative Cobb angle in neutral $(\mathrm{p}=0.000)$, larger degree of preoperative flexion $(p=0.023)$, and small degree of preoperative extension ( $p$ $=0.025)$ were all important predictors. ${ }^{62}$

As demonstrated by 2 studies, an increase in the number of operative lamina is significantly associated with an increase incidence of axial pain $(\mathrm{p}<0.001, \mathrm{p}=0.03) .^{54,61}$ A diagnosis of OPLL (compared with CSM) is also an important clinical predictor of axial pain $(\mathrm{p}=0.027) .{ }^{54}$

As indicated by single studies, risk factors for various surgical complications include 1$)$ younger age $(\mathrm{p}<0.05)$ and multilevel surgery $(\mathrm{p}<0.002)$ for pseudarthrosis,$\left.{ }^{16} 2\right)$ previous surgery for graft complications $\left.(\mathrm{p}<0.001),{ }^{16} 3\right)$ preoperative kyphosis for closure of lamina $(\mathrm{p}=0.014),{ }^{51}$ and 4) a larger number of fused segments for reconstruction failure $(4.2$ vs $3.5, \mathrm{p}<0.05) .56$ Age $(\mathrm{n}=3)^{51,56,68}$ and sex $(n=3)^{51,56,68}$ were not significant predictors of surgical complications.

\section{KQ2: Are Rates of Complications Different Between Surgical Interventions or Varying Techniques? \\ Anterior Versus Posterior}

Fehlings et al. compared rates of complications between anterior and posterior surgical groups. ${ }^{18,19}$ The patient demographics were significantly different in the 2 cohorts: patients treated anteriorly were on average younger, their condition was less severe, they had fewer operated levels, and experienced a smaller volume of blood loss than those treated posteriorly. There were, however, no significant differences in rates of major and/or minor complications $(\mathrm{p}=$ $0.11^{19}$ and $\left.\mathrm{p}=0.197^{18}\right)$, major complications $(\mathrm{p}=0.61)$, new neurological deficits $(\mathrm{p}=1.00), C-5$ radiculopathy $(\mathrm{p}=1.00)$, or dysphagia $(p=0.65)$. Based on results from Fehlings et al., there was a higher incidence of postoperative infection in the posterior group (4.7\%) compared with the anterior group $(0.6 \%, \mathrm{p}=0.030)$. Ghogawala et al. also reported similar overall complication rates $(\mathrm{p}=1.00)$ between patients who underwent anterior cervical decompression and fusion and those treated posteriorly by laminectomy with fixation. ${ }^{22}$ In addition, there were no significant differences in rates of overall $(p=0.654)$, surgical $(p=0.507)$, medical $(\mathrm{p}=1.00)$, or irreversible complications $(\mathrm{p}=0.74)$ between corpectomy and laminectomy and fusion surgeries. ${ }^{44}$ The frequency of dysphagia and transient hoarseness, however, was greater in the corpectomy group (7.1\%) compared with the laminectomy and fusion group $(0 \%)$.

Five studies analyzed differences in complication rates between anterior decompression surgery and laminoplasty. 15,30,42,48,59 The surgical approach was at the discretion of the attending surgeon in 2 studies. ${ }^{15,48}$ In the other 3 studies, the design was quasi-randomized: patients were treated anteriorly within a specific time frame and posteriorly in subsequent years. ${ }^{30,42,59}$ Based on results from Edwards et al. and Liu et al., there was a higher incidence of complications in the anterior group (ACDF with plate cage Benezech [PCB; $p<0.05]$ or corpectomy [p < 0.05]) compared with laminoplasty group. The rates of axial pain were found to be significantly higher in the laminoplasty group as compared to the ADF group. ${ }^{30}$ These conclusions, however, were based on single studies. According to Sakaura et al., there were no significant differences in rates of C-5 palsy, bone graft complications, donor site morbidities, axial pain, or postoperative kyphosis between the groups. ${ }^{59}$

\section{Anterior Techniques}

Three studies compared the efficacy and safety of various anterior approaches: ACDF versus Bryan disc pros- 







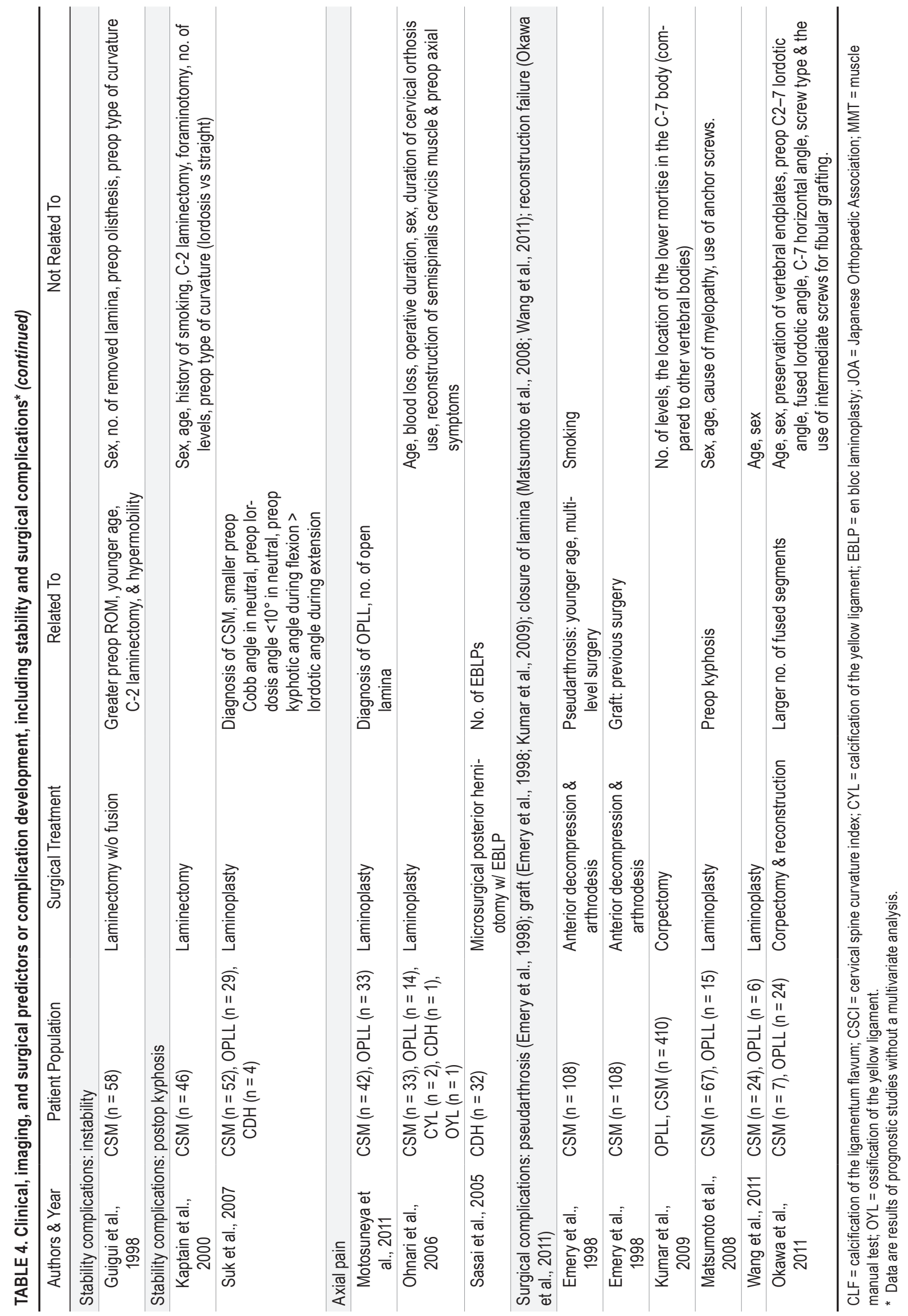


thesis $(n=1)^{11}$ and ADCF versus corpectomy $(n=2) .{ }^{47,63}$ Cheng et al. conducted a randomized control trial to compare operative and postoperative characteristics between a Bryan disc and an ACDF group. ${ }^{11}$ Complication rates were higher in the ACDF group since dysphagia was seen in 7 patients as compared to only 1 in the Bryan group ( $\mathrm{p}<$ 0.001).

When comparing ACDF to anterior corpectomy, there were no significant differences in rates of surgical complications ( $p=0.694)$, CSF $(p=0.604)$, hoarseness (Song et al., $p=0.742^{63}$; Lin et al., $\left.p=1.00^{47}\right)$, epidural hematoma $(\mathrm{p}=1.00), \mathrm{C}-5$ radiculopathy $(\mathrm{p}=1.00)$, dysphagia (Song et al., $\mathrm{p}=0.436$; Lin et al., $\mathrm{p}=1.00)$, graft dislodgement $(\mathrm{p}=0.246)$, subsidence $(\mathrm{p}=0.121)$, and dural tear $(\mathrm{p}=$ 0.688). Although graft-related complications were not significantly different between surgical groups in the study by Song et al., Lin et al. reported a higher incidence of total instrumented and graft-related complications in the corpectomy group $(\mathrm{p}=0.032)$.

\section{Posterior Techniques}

Four studies compared complication rates between laminoplasty and laminectomy and fusion. ${ }^{10,19,70,72}$ According to studies by Fehlings et al. and Woods et al., there is no difference in overall complication rates between posterior surgeries (Fehlings et al., $\mathrm{p}=0.14$; Woods et al., $\mathrm{p}=$ 0.087). In addition, rates of wound infections (Yang et al., $p=0.285$; Fehlings et al., $p=0.43)$, dysphagia $(p=0.32)$, neck pain $(\mathrm{p}=0.066)$, CSF leakage $(\mathrm{p}=0.252)$, kyphosis $(\mathrm{p}=0.756)$, and restenosis $(\mathrm{p}=0.448)$ were not significantly different between the 2 groups. In studies by Chen et al. and Yang et al., there was a higher incidence of C-5 palsy in the laminectomy and fusion group compared with the laminoplasty group $(\mathrm{p}<0.05)$. Fehlings et al., however, noted no differences in $\mathrm{C}-5$ radiculopathy rates between the 2 posterior techniques. Although Chen et al. reported no significant differences between laminoplasty and laminectomy and fusion with respect to axial pain, Yang et al. found a higher incidence of axial pain in patients treated with laminectomy and fusion.

\section{Laminoplasty Techniques}

Various laminoplasty techniques have been designed and modified to improve surgical outcome and decrease postoperative morbidity. In this review, 11 studies compared complication rates between different forms of laminoplasty procedures. In a quasi-randomized study, Park et al. observed no significant differences in aggravated neck pain $(\mathrm{p}=0.34), \mathrm{C}-5$ palsy $(\mathrm{p}=0.34)$, or other complications $(\mathrm{p}=1.00)$ between midline-splitting and unilateral single-door laminoplasty. ${ }^{57}$ Similarly, hinge position (wide group: hinge located at the inner margin of the lateral mass; narrow group: hinge at the lamina margin) was not associated with the development of $\mathrm{C}-5$ palsy $(\mathrm{p}=0.17){ }^{71}$ In a comparative study between open-door and doubledoor laminoplasty, C-5 palsy rates tended to be higher following open-door laminoplasty $(\mathrm{p}<0.05) .{ }^{34}$

Selective expansive open-door laminoplasty (EOLP) was designed to reduce damage to cervical posterior elements and prevent common long-term problems of laminoplasty, such as axial symptoms, malalignment, and decreased range of motion. ${ }^{66}$ This procedure consists of an open-door laminoplasty at the levels of stenosis and a partial laminectomy combined with laminoplasty at the most inferior stenosis level. As indicated by Tsuji et al., the incidence of C-5 palsy was significantly lower in the selective EOLP group than in a C3-7 EOLP group ( $p=0.037)$.

Another modification of laminoplasty was designed to reduce the amount of bone graft during operation (3 levels only in the open side), shorten the period of orthosis application, and encourage patients to exercise their posterior neck muscles postoperatively. ${ }^{39}$ Complication rates in this modified group were compared with rates following traditional open-door en bloc laminoplasty. ${ }^{32}$ There was a lower incidence of axial pain and neck stiffness in the modified group $(\mathrm{p}=0.0019) .{ }^{39}$

Two studies examined the effectiveness of foraminotomy in combination with laminoplasty in preventing postoperative C-5 palsy. ${ }^{38,43}$ Both Komagata et al. and Katsumi et al. reported a significant lower incidence of C-5 palsy in the concurrent foraminotomy group $(\mathrm{p}<0.05)$.

Four studies have explored muscle-preserving techniques and have compared rates of axial pain, postoperative kyphosis, and C-5 nerve root palsy. ${ }^{28,29,58,60}$ Sakaura et al. examined whether preservation of 1) subaxial deep extensor muscles or 2) the funicular section of the nuchal ligament attached to the C-6 and C-7 spinous process could prevent poor radiological outcomes and axial pain. Based on results from these 2 studies, there was no significant difference in rates of axial pain between muscle-preserving and muscle-disrupting groups. Incidence of postoperative kyphosis was not different between the subaxial deep extensor preserving group and the control group.

The cohort study conducted by Hosono et al. compared C3-7 to C3-6 laminoplasty. The only difference between the procedures was that the $\mathrm{C}-7$ lamina was opened in the C3-7 group because these patients exhibited cord compression at C-6/C-7 or lower levels. Early and late axial pain rates were higher in the $\mathrm{C} 3-7$ group, likely due to disruption of the trapezius and rhomboideus minor muscles that typically attach to the C-7 vertebra (early: $\mathrm{p}=$ 0.006; late: $p=0.015$ ). These results were confirmed by a second study by Hosono et al.: there was a higher incidence of early and late axial pain in the left-opened C3-7 group compared with either the left or right opened C3-6 groups (early: $\mathrm{p}=0.0008$; late: $\mathrm{p}=0.0036$ ). These studies are summarized in Table 5.

\section{Summary of Evidence}

Table 6 describes how the overall quality of evidence was derived. High evidence suggests that old age is predictive of perioperative complications. Low evidence reports old age decreases the risk of postoperative axial pain. Age, however, is not associated with upper extremity/C-5 palsy (low evidence).

Based on low evidence, there is no association between comorbidities, baseline severity score, BMI, duration of symptoms and smoking status, and perioperative complications. Sex is also not a significant predictor (moderate evidence).

Low evidence suggests that patients with a diagnosis of 


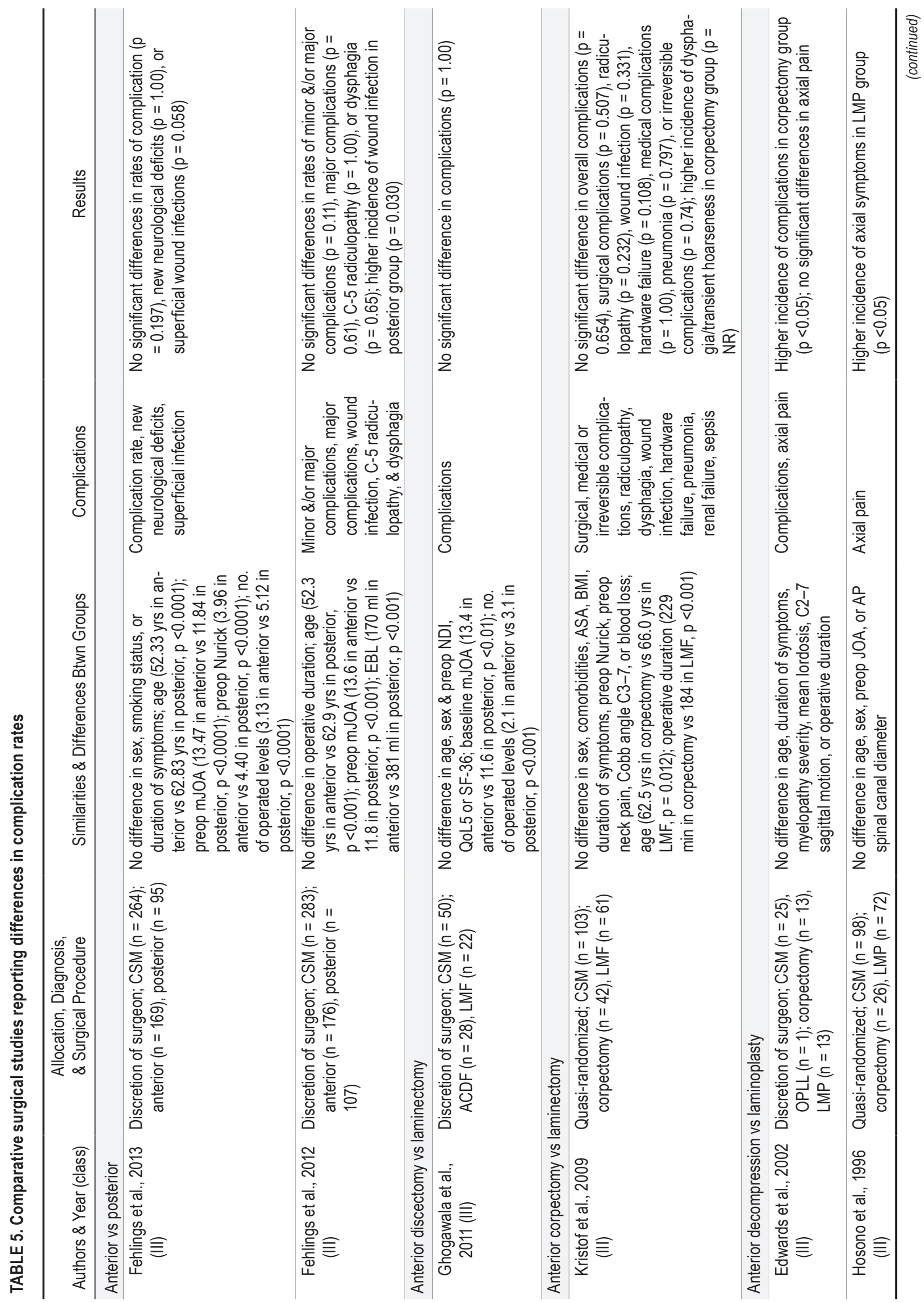




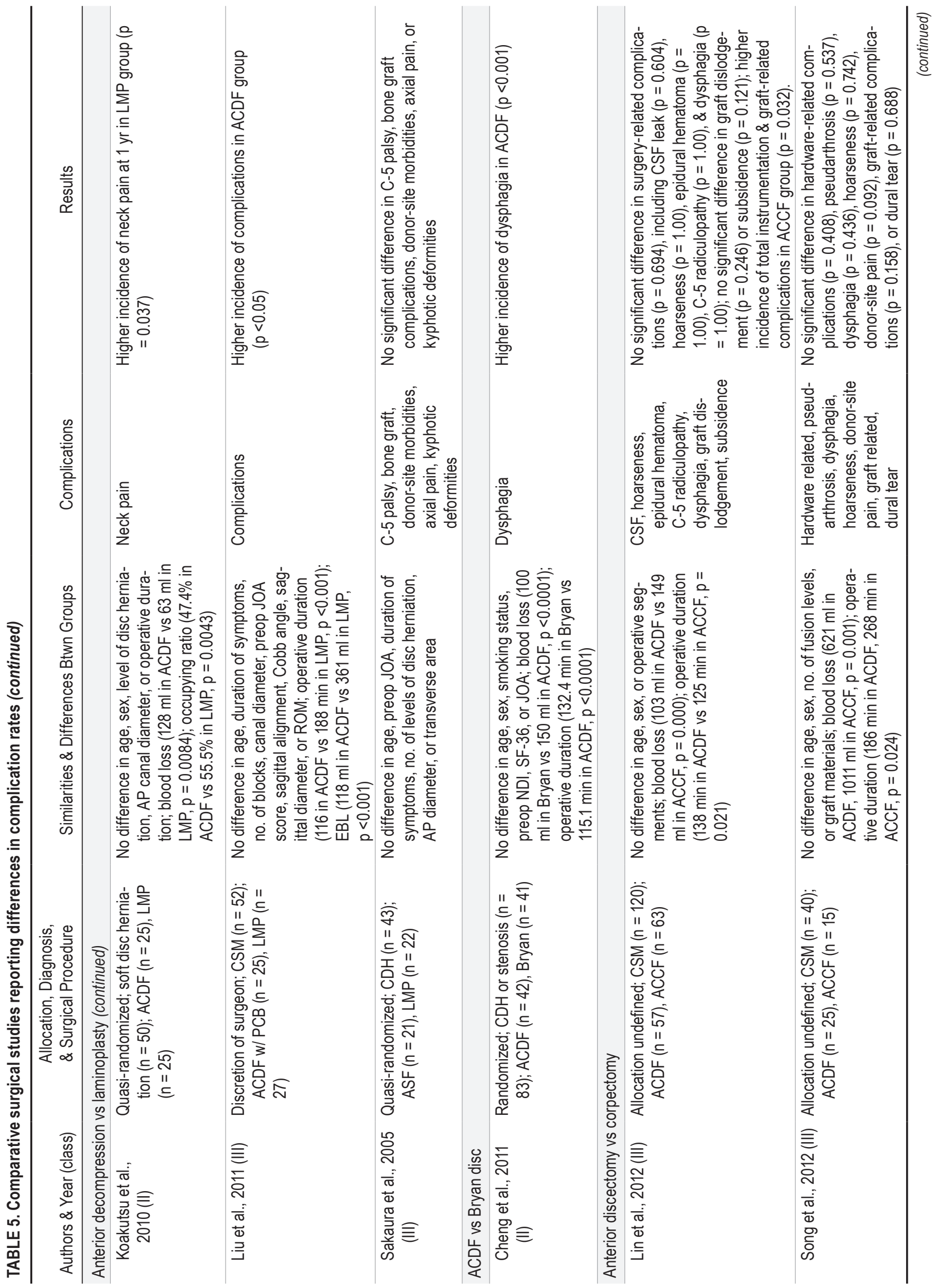




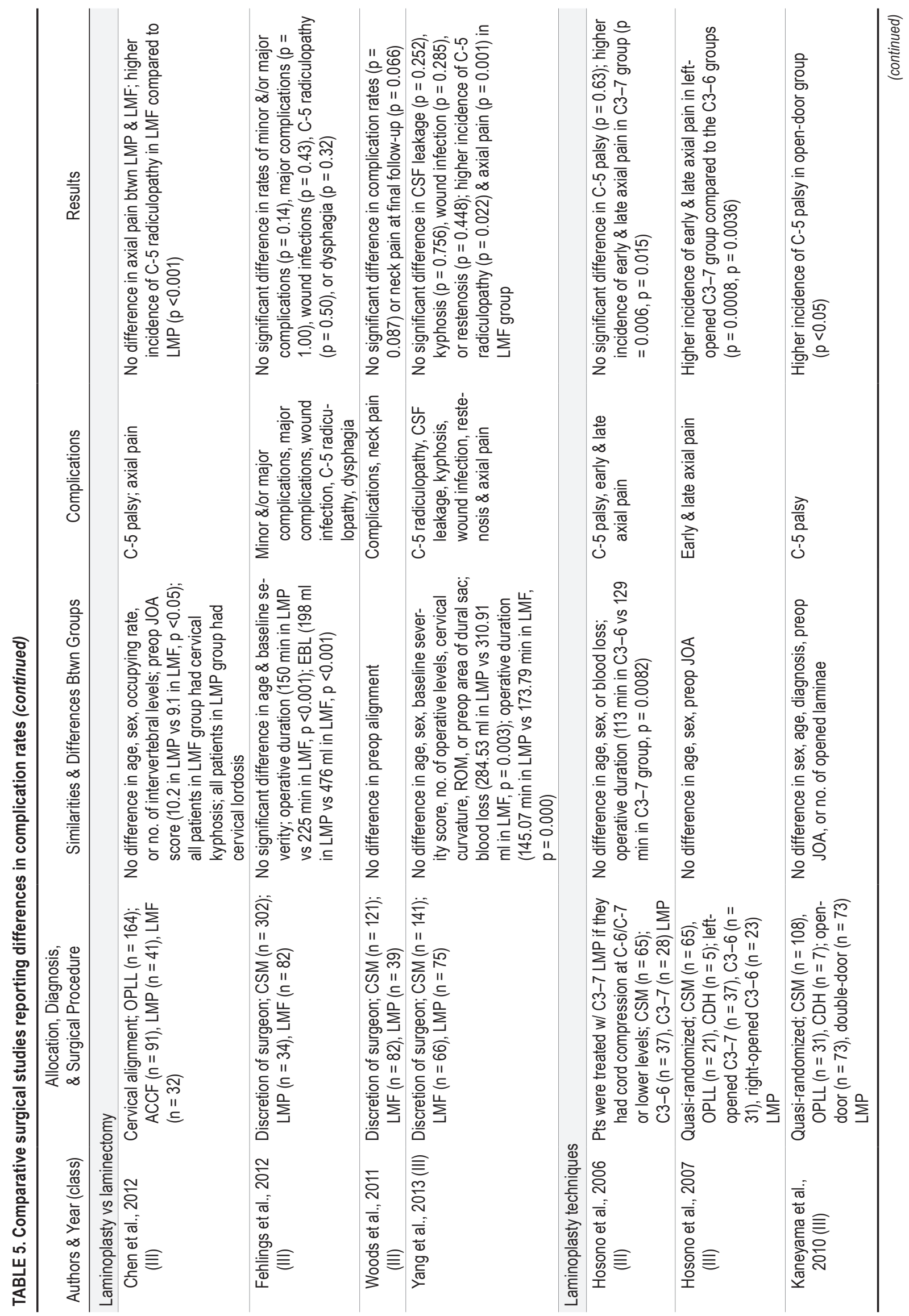




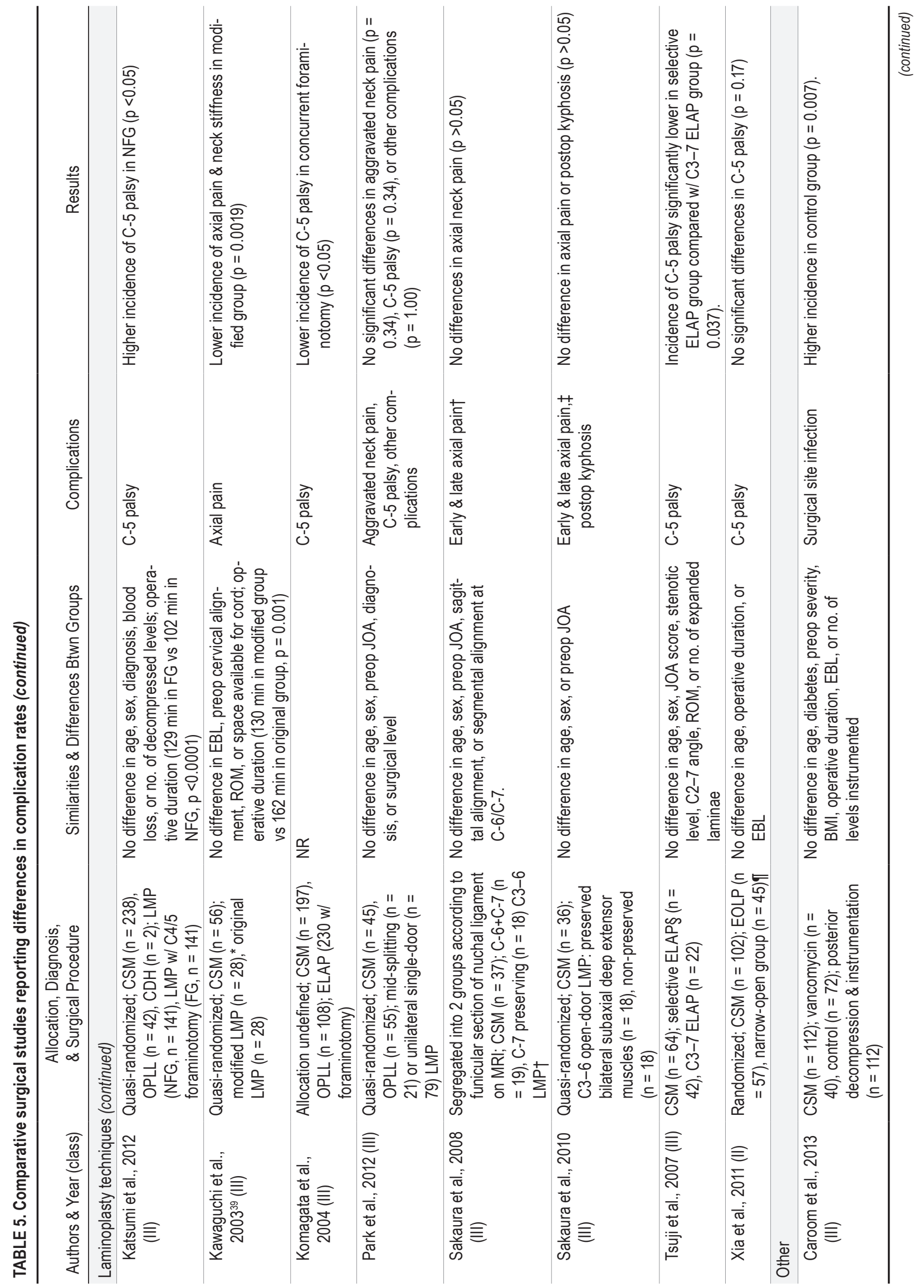


OPLL are at a greater risk of developing upper extremity palsy or C-5 palsy postoperatively.

Low to moderate evidence suggests no association between surgical approach, number of levels, estimated blood loss, laminoplasty and corpectomy, and perioperative complications. Longer operative duration is predictive of overall perioperative complications (moderate evidence) but not of major complications (low evidence).

Based on moderate evidence, there are higher rates of neck pain in laminoplasty compared with anterior spinal fusion and higher rates of dysphagia in ACDF than in Bryan disc. With respect to laminoplasty techniques, there is no difference in rates of C-5 palsy between wide and open-door laminoplasty (moderate evidence). Low evidence reports a lower incidence of C-5 palsy in laminoplasty with concurrent foraminotomy compared with no foraminotomy.

\section{Discussion}

This review summarizes important clinical and surgical predictors of postoperative complications. This knowledge will allow clinicians to identify high-risk patients and institute rigorous prevention strategies. Furthermore, surgeons can use this information to objectively discuss surgical risks with their patients. This, together with outcome prediction, will enable patients to make more informed decisions during the surgical-consent process and will also aid in expectation management. Finally, from an economic standpoint, health care providers will be able to appropriately allocate resources and predict future hospital utilization costs for each surgical patient.

Based on this review, age is a significant predictor of postoperative complications. This is consistent with previous lumbar spine studies that have shown significant adverse events in elderly patients following surgery. ${ }^{5,14}$ As CSM is a progressive disease, older patients are likely to have substantial degenerative pathology and will require a more complex surgery. ${ }^{19}$ In addition, the elderly can be less tolerant to surgery due to poorer overall general health status, comorbidities, and reduced physiological reserves.

There is controversy in the literature surrounding the predictive value of comorbidities. The number of comorbidities was significantly associated with perioperative complications in a study by Boakye et al. ${ }^{3}$ This finding was based on a large retrospective analysis of 58,115 patients undergoing spinal fusion at a single hospital. Furlan et al., on the other hand, reported no relationship between the number of ICD-9 codes and perioperative complications. ${ }^{21}$ This insignificant multivariate association may be due to a potential interaction between older age and number of comorbidities. Patients with diabetes, particularly uncontrolled or Type I, are at a greater risk of perioperative complications and may require a longer length of stay and more hospital resources. These findings are consistent with results from previous lumbar spine studies that concluded diabetes is a significant risk factor of wound infections and nonunions. , 17,23,62 $^{2}$

With respect to surgical factors, a longer operative duration is associated with greater perioperative complication rates. Duration of surgery may be a surrogate for case 


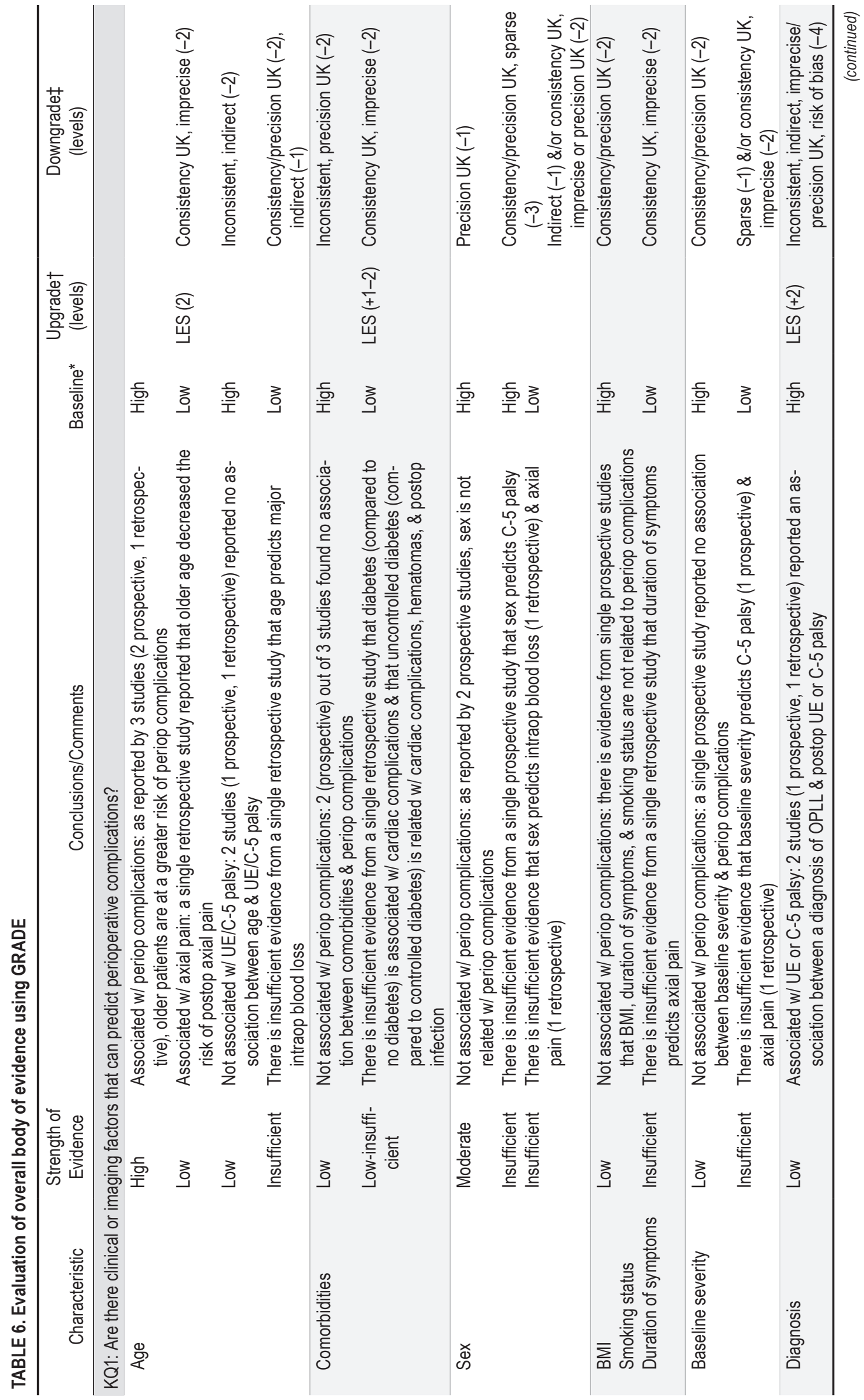




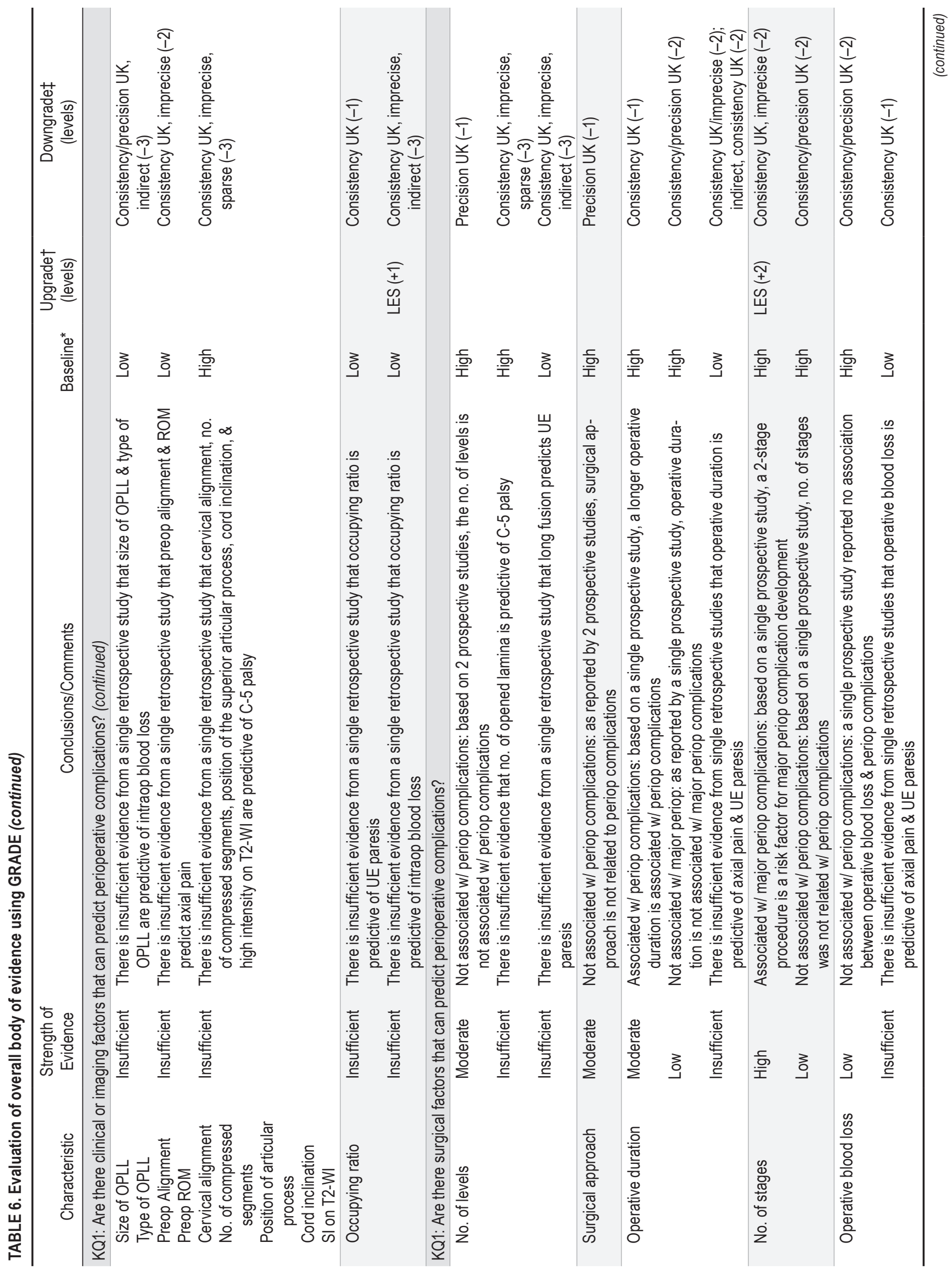




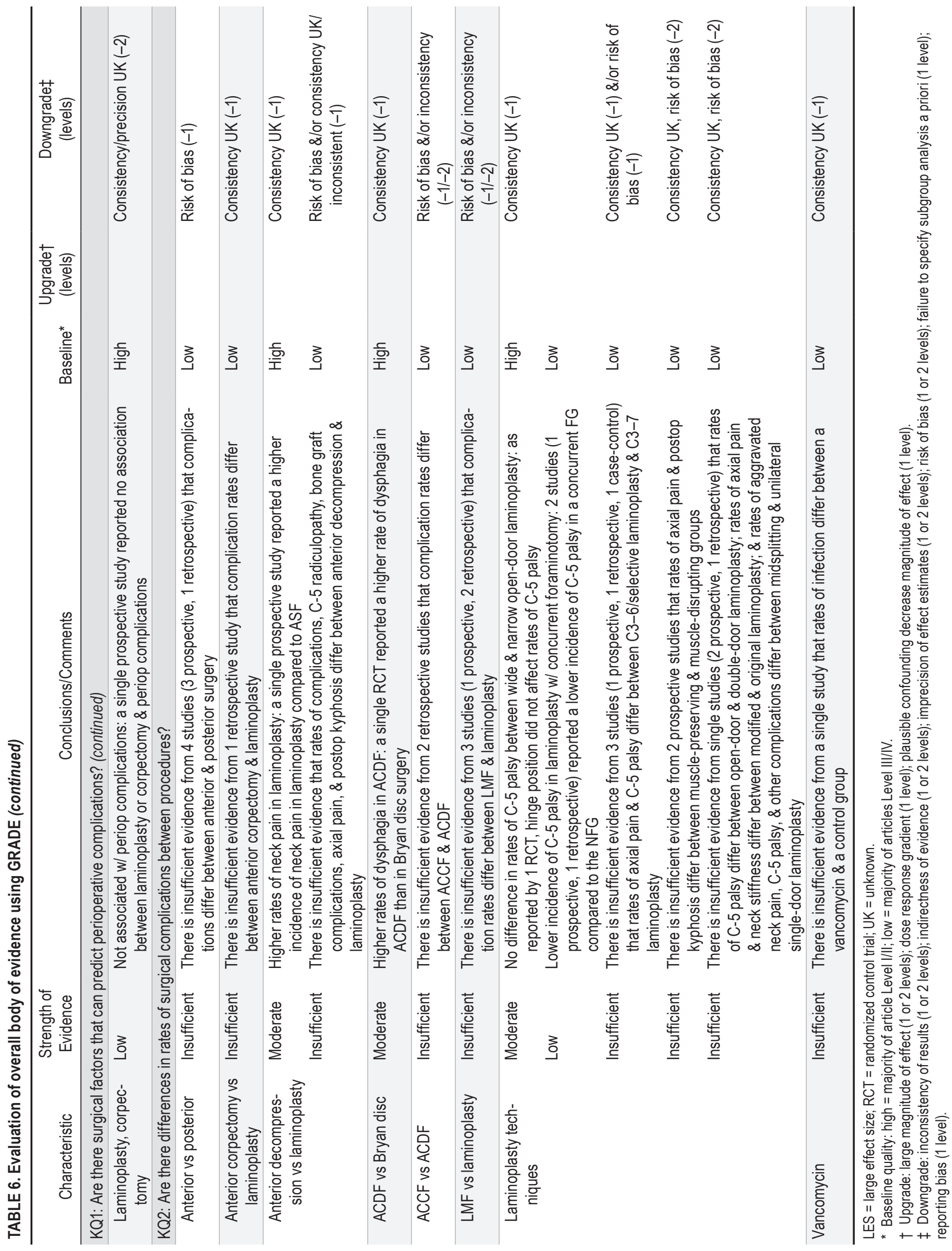


complexity. Therefore, we do not recommend surgeons speed up surgery to prevent complications but rather identify complex cases, anticipate complications and plan accordingly. A 2-stage anteroposterior surgery is predictive of major complications; similarly, this factor may reflect greater degenerative pathology and increased case complexity.

The second objective of this review was to compare complication rates between various surgical techniques. Based on the results of KQ1, studies had to control for age and operative duration to be classified as good quality cohort studies. Unfortunately, the majority of the literature included in this review did not adjust for these 2 confounders, preventing accurate comparisons between groups.

There was low to moderate evidence, however, suggesting differences in complication rates between ACDF and laminoplasty, ACDF and Bryan's disc, narrow and widehinge laminoplasty, and presence and absence of concurrent foraminotomy. Postoperative neck pain is frequently encountered following laminoplasty and rates are significantly higher than following anterior spinal fusion.

Current hypotheses for intra- or postoperative C-5 nerve root palsy include traumatic surgical procedure, edema of the spinal cord, or tethering of the root. Yonenobu et al. suggested that C-5 palsy is more common following wideopen door laminoplasty due to excessive backward shift of the spinal cord. ${ }^{73,74}$ The hinge position determines how open the door is and an inward shift of the hinge restricts excessive backward shift and overstretching of the nerve roots. ${ }^{71}$ Although no patients in the narrow-group experienced C-5 palsy and 3 cases were seen in the wide group, this difference did not reach statistical significance. However, there was a lower incidence of this complication following laminoplasty with concurrent foraminotomy, suggesting that another potential cause of C-5 palsy is existing C-4/C-5 foraminal stenosis. ${ }^{38}$

\section{Strengths and Limitations}

This is the first systematic review exploring important clinical, imaging, and surgical predictors of complications. It has highlighted some of the key knowledge gaps in the literature, including a lack of high-quality prospective prognostic studies and poor control of confounding variables in comparative studies. Our study has its limitations. First, our search was restricted to studies published in English, and as a result, some relevant abstracts were excluded. Second, although we analyzed the studies based on type of complication, we could not separate based on timing of complications. Third, articles that dichotomized a predictor might have done it differently (e.g., age). Finally, since the review process and critical appraisal does require scientific judgment, there is potential for discrepancy among reviewers and bias.

\section{Evidence-Based Clinical Recommendations}

Recommendation 1: While surgeons should not discriminate on the basis of age, they should be informed that older patients are at a higher risk of complications and are less likely to achieve a modified Japanese Orthopaedic Association (JOA) score $\geq 16$. We therefore recommend surgeons discuss these risks with their patients, plan and institute rigorous preventative strategies, and closely monitor their patients in the perioperative period. Furthermore, health care systems should anticipate higher associated costs and allocate resources accordingly.

\section{Strength of Statement: Moderate}

Recommendation 2: Longer operative duration and 2-stage surgery are important predictors of complications. However, both likely reflect substantial degenerative pathology and increased case complexity. We therefore recommend surgeons identify these cases preoperatively, anticipate complications, and plan both preventative and postoperative management strategies.

\section{Strength of Statement: Moderate}

Consensus Statement 1: We suggest that results from this study guide the development of future complication prediction rules. Furthermore, we suggest that analyses in comparative complication studies control for operative duration and age.

\section{References}

1. Atkins D, Best D, Briss PA, Eccles M, Falck-Ytter Y, Flottorp $\mathrm{S}$, et al: Grading quality of evidence and strength of recommendations. BMJ 328:1490, 2004

2. Bendo JA, Spivak J, Moskovich R, Neuwirth M: Instrumented posterior arthrodesis of the lumbar spine in patients with diabetes mellitus. Am J Orthop 29:617-620, 2000

3. Boakye M, Patil CG, Santarelli J, Ho C, Tian W, Lad SP: Cervical spondylotic myelopathy: complications and outcomes after spinal fusion. Neurosurgery 62:455-462, 2008

4. Caroom C, Tullar JM, Benton EG Jr, Jones JR, Chaput CD: Intrawound vancomycin powder reduces surgical site infections in posterior cervical fusion. Spine (Phila Pa 1976) 38:1183-1187, 2013

5. Carreon LY, Puno RM, Dimar JR II, Glassman SD, Johnson JR: Perioperative complications of posterior lumbar decompression and arthrodesis in older adults. J Bone Joint Surg Am 85-A:2089-2092, 2003

6. Casha S, Engelbrecht HA, DuPlessis SJ, Hurlbert RJ: Suspended laminoplasty for wide posterior cervical decompression and intradural access: results, advantages, and complications. J Neurosurg Spine 1:80-86, 2004

7. Chen Y, Chen D, Wang X, Guo Y, He Z: C5 palsy after laminectomy and posterior cervical fixation for ossification of posterior longitudinal ligament. J Spinal Disord Tech 20:533-535, 2007

8. Chen Y, Chen D, Wang X, Yang H, Liu X, Miao J, et al: Significance of segmental instability in cervical ossification of the posterior longitudinal ligament and treated by a posterior hybrid technique. Arch Orthop Trauma Surg 133:171-177, 2013

9. Chen Y, Guo Y, Chen D, Wang X, Lu X, Yuan W: Long-term outcome of laminectomy and instrumented fusion for cervical ossification of the posterior longitudinal ligament. Int Orthop 33:1075-1080, 2009

10. Chen Y, Liu X, Chen D, Wang X, Yuan W: Surgical strategy for ossification of the posterior longitudinal ligament in the cervical spine. Orthopedics 35:e1231-e1237, 2012

11. Cheng L, Nie L, Li M, Huo Y, Pan X: Superiority of the Bryan ${ }^{\circledR}$ disc prosthesis for cervical myelopathy: a randomized study with 3-year followup. Clin Orthop Relat Res 469:3408-3414, 2011

12. Chiba K, Toyama Y, Matsumoto M, Maruiwa H, Watanabe M, Hirabayashi K: Segmental motor paralysis after expansive 
open-door laminoplasty. Spine (Phila Pa 1976) 27:21082115,2002

13. Cook C, Tackett S, Shah A, Pietrobon R, Browne J, Viens N, et al: Diabetes and perioperative outcomes following cervical fusion in patients with myelopathy. Spine (Phila Pa 1976) 33:E254-E260, 2008 [Erratum in Spine (Phila Pa 1976) 34:2470-2472, 2009]

14. Deyo RA, Cherkin DC, Loeser JD, Bigos SJ, Ciol MA: Morbidity and mortality in association with operations on the lumbar spine. The influence of age, diagnosis, and procedure. J Bone Joint Surg Am 74:536-543, 1992

15. Edwards CC II, Heller JG, Murakami H: Corpectomy versus laminoplasty for multilevel cervical myelopathy: an independent matched-cohort analysis. Spine (Phila Pa 1976) 27:1168-1175, 2002

16. Emery SE, Bohlman HH, Bolesta MJ, Jones PK: Anterior cervical decompression and arthrodesis for the treatment of cervical spondylotic myelopathy. Two to seventeen-year follow-up. J Bone Joint Surg Am 80:941-951, 1998

17. Fang A, Hu SS, Endres N, Bradford DS: Risk factors for infection after spinal surgery. Spine (Phila Pa 1976) 30:14601465,2005

18. Fehlings MG, Barry S, Kopjar B, Yoon ST, Arnold P, Massicotte EM, et al: Anterior versus posterior surgical approaches to treat cervical spondylotic myelopathy: outcomes of the prospective multicenter AOSpine North America CSM study in 264 patients. Spine (Phila Pa 1976) 38:2247-2252, 2013

19. Fehlings MG, Smith JS, Kopjar B, Arnold PM, Yoon ST, Vaccaro AR, et al: Perioperative and delayed complications associated with the surgical treatment of cervical spondylotic myelopathy based on 302 patients from the AOSpine North America Cervical Spondylotic Myelopathy Study. J Neurosurg Spine 16:425-432, 2012

20. Fehlings MG, Wilson JR, Kopjar B, Yoon ST, Arnold PM, Massicotte EM, et al: Efficacy and safety of surgical decompression in patients with cervical spondylotic myelopathy: results of the AOSpine North America prospective multicenter study. J Bone Joint Surg Am 95:1651-1658, 2013

21. Furlan JC, Kalsi-Ryan S, Kailaya-Vasan A, Massicotte EM, Fehlings MG: Functional and clinical outcomes following surgical treatment in patients with cervical spondylotic myelopathy: a prospective study of 81 cases. J Neurosurg Spine 14:348-355, 2011

22. Ghogawala Z, Martin B, Benzel EC, Dziura J, Magge SN, Abbed KM, et al: Comparative effectiveness of ventral vs dorsal surgery for cervical spondylotic myelopathy. Neurosurgery 68:622-631, 2011

23. Glassman SD, Alegre G, Carreon L, Dimar JR, Johnson JR: Perioperative complications of lumbar instrumentation and fusion in patients with diabetes mellitus. Spine J 3:496-501, 2003

24. Greiner-Perth R, Elsaghir H, Böhm H, El-Meshtawy M: The incidence of C5-C6 radiculopathy as a complication of extensive cervical decompression: own results and review of literature. Neurosurg Rev 28:137-142, 2005

25. Guigui P, Benoist M, Deburge A: Spinal deformity and instability after multilevel cervical laminectomy for spondylotic myelopathy. Spine (Phila Pa 1976) 23:440-447, 1998

26. Hasegawa K, Homma T, Chiba Y: Upper extremity palsy following cervical decompression surgery results from a transient spinal cord lesion. Spine (Phila Pa 1976) 32:E197E202, 2007

27. Holly LT, Moftakhar P, Khoo LT, Shamie AN, Wang JC: Surgical outcomes of elderly patients with cervical spondylotic myelopathy. Surg Neurol 69:233-240, 2008

28. Hosono N, Sakaura H, Mukai Y, Fujii R, Yoshikawa H: C3-6 laminoplasty takes over C3-7 laminoplasty with significantly lower incidence of axial neck pain. Eur Spine J 15:13751379,2006
29. Hosono N, Sakaura H, Mukai Y, Yoshikawa H: The source of axial pain after cervical laminoplasty-C7 is more crucial than deep extensor muscles. Spine (Phila Pa 1976) 32:29852988, 2007

30. Hosono N, Yonenobu K, Ono K: Neck and shoulder pain after laminoplasty. A noticeable complication. Spine (Phila Pa 1976) 21:1969-1973, 1996

31. Imagama S, Matsuyama Y, Yukawa Y, Kawakami N, Kamiya M, Kanemura T, et al: C5 palsy after cervical laminoplasty: a multicentre study. J Bone Joint Surg Br 92:393-400, 2010

32. Itoh T, Tsuji H: Technical improvements and results of laminoplasty for compressive myelopathy in the cervical spine. Spine (Phila Pa 1976) 10:729-736

33. Kalsi-Ryan S, Karadimas SK, Fehlings MG: Cervical spondylotic myelopathy: the clinical phenomenon and the current pathobiology of an increasingly prevalent and devastating disorder. Neuroscientist 19:409-421, 2013

34. Kaneyama S, Sumi M, Kanatani T, Kasahara K, Kanemura A, Takabatake M, et al: Prospective study and multivariate analysis of the incidence of C5 palsy after cervical laminoplasty. Spine (Phila Pa 1976) 35:E1553-E1558, 2010

35. Kaptain GJ, Simmons NE, Replogle RE, Pobereskin L: Incidence and outcome of kyphotic deformity following laminectomy for cervical spondylotic myelopathy. J Neurosurg 93 (2 Suppl):199-204, 2000

36. Kato M, Nakamura H, Konishi S, Dohzono S, Toyoda H, Fukushima W, et al: Effect of preserving paraspinal muscles on postoperative axial pain in the selective cervical laminoplasty. Spine (Phila Pa 1976) 33:E455-E459, 2008

37. Kato S, Chikuda H, Seichi A, Ohtsu H, Kimura A, Toyama Y: Radiographical risk factors for major intraoperative blood loss during laminoplasty in patients with ossification of the posterior longitudinal ligament. Spine (Phila Pa 1976) 37:E1588-E1593, 2012

38. Katsumi K, Yamazaki A, Watanabe K, Ohashi M, Shoji H: Can prophylactic bilateral C4/C5 foraminotomy prevent postoperative C5 palsy after open-door laminoplasty?: a prospective study. Spine (Phila Pa 1976) 37:748-754, 2012

39. Kawaguchi Y, Kanamori M, Ishiara H, Nobukiyo M, Seki S, Kimura T: Preventive measures for axial symptoms following cervical laminoplasty. J Spinal Disord Tech 16:497-501, 2003

40. Kawaguchi Y, Kanamori M, Ishihara H, Ohmori K, Abe Y, Kimura T: Pathomechanism of myelopathy and surgical results of laminoplasty in elderly patients with cervical spondylosis. Spine (Phila Pa 1976) 28:2209-2214, 2003

41. Kimura A, Seichi A, Hoshino Y, Yamazaki M, Mochizuki M, Aiba A, et al: Perioperative complications of anterior cervical decompression with fusion in patients with ossification of the posterior longitudinal ligament: a retrospective, multi-institutional study. J Orthop Sci 17:667-672, 2012

42. Koakutsu T, Morozumi N, Ishii Y, Kasama F, Sato T, Tanaka $Y$, et al: Anterior decompression and fusion versus laminoplasty for cervical myelopathy caused by soft disc herniation: a prospective multicenter study. J Orthop Sci 15:71-78, 2010

43. Komagata M, Nishiyama M, Endo K, Ikegami H, Tanaka S, Imakiire A: Prophylaxis of C5 palsy after cervical expansive laminoplasty by bilateral partial foraminotomy. Spine $\mathbf{J}$ 4:650-655, 2004

44. Kristof RA, Kiefer T, Thudium M, Ringel F, Stoffel M, Kovacs A, et al: Comparison of ventral corpectomy and platescrew-instrumented fusion with dorsal laminectomy and rod-screw-instrumented fusion for treatment of at least two vertebral-level spondylotic cervical myelopathy. Eur Spine J 18:1951-1956, 2009

45. Kumar GS, Rajshekhar V: Acute graft extrusion following central corpectomy in patients with cervical spondylotic myelopathy and ossified posterior longitudinal ligament. J Clin Neurosci 16:373-377, 2009 
46. Lawrence BD, Shamji MF, Traynelis VC, Yoon ST, Rhee JM, Chapman JR, et al: Surgical management of degenerative cervical myelopathy: a consensus statement. Spine (Phila Pa 1976) 38 (22 Suppl 1):S171-S172, 2013

47. Lin Q, Zhou X, Wang X, Cao P, Tsai N, Yuan W: A comparison of anterior cervical discectomy and corpectomy in patients with multilevel cervical spondylotic myelopathy. Eur Spine J 21:474-481, 2012

48. Liu T, Yang HL, Xu YZ, Qi RF, Guan HQ: ACDF with the PCB cage-plate system versus laminoplasty for multilevel cervical spondylotic myelopathy. J Spinal Disord Tech 24:213-220, 2011

49. Liu T, Zou W, Han Y, Wang Y: Correlative study of nerve root palsy and cervical posterior decompression laminectomy and internal fixation. Orthopedics 33:33, 2010

50. Lu J, Wu X, Li Y, Kong X: Surgical results of anterior corpectomy in the aged patients with cervical myelopathy. Eur Spine J 17:129-135, 2008

51. Matsumoto M, Watanabe K, Tsuji T, Ishii K, Takaishi H, Nakamura M, et al: Risk factors for closure of lamina after open-door laminoplasty. J Neurosurg Spine 9:530-537, 2008

52. Meyer SA, Wu JC, Mummaneni PV: Laminoplasty outcomes: is there a difference between patients with degenerative stenosis and those with ossification of the posterior longitudinal ligament? Neurosurg Focus 30(3):E9, 2011

53. Minoda Y, Nakamura H, Konishi S, Nagayama R, Suzuki E, Yamano Y, et al: Palsy of the C5 nerve root after midsagittalsplitting laminoplasty of the cervical spine. Spine (Phila Pa 1976) 28:1123-1127, 2003

54. Motosuneya T, Maruyama T, Yamada H, Tsuzuki N, Sakai H: Long-term results of tension-band laminoplasty for cervical stenotic myelopathy: a ten-year follow-up. J Bone Joint Surg Br 93:68-72, 2011

55. Ohnari H, Sasai K, Akagi S, Iida H, Takanori S, Kato I: Investigation of axial symptoms after cervical laminoplasty, using questionnaire survey. Spine J 6:221-227, 2006

56. Okawa A, Sakai K, Hirai T, Kato T, Tomizawa S, Enomoto $\mathrm{M}$, et al: Risk factors for early reconstruction failure of multilevel cervical corpectomy with dynamic plate fixation. Spine (Phila Pa 1976) 36:E582-E587, 2011

57. Park JH, Roh SW, Rhim SC, Jeon SR: Long-term outcomes of 2 cervical laminoplasty methods: midline splitting versus unilateral single door. J Spinal Disord Tech 25:E224-E229, 2012

58. Sakaura H, Hosono N, Mukai Y, Fujimori T, Iwasaki M, Yoshikawa H: Preservation of muscles attached to the $\mathrm{C} 2$ and C7 spinous processes rather than subaxial deep extensors reduces adverse effects after cervical laminoplasty. Spine (Phila Pa 1976) 35:E782-E786, 2010

59. Sakaura H, Hosono N, Mukai Y, Ishii T, Iwasaki M, Yoshikawa H: Long-term outcome of laminoplasty for cervical myelopathy due to disc herniation: a comparative study of laminoplasty and anterior spinal fusion. Spine (Phila Pa 1976) 30:756-759, 2005

60. Sakaura H, Hosono N, Mukai Y, Oshima K, Iwasaki M, Yoshikawa H: Preservation of the nuchal ligament plays an important role in preventing unfavorable radiologic changes after laminoplasty. J Spinal Disord Tech 21:338-343, 2008

61. Sasai K, Saito T, Ohnari H, Yamamoto T, Kasuya T, Wakabayashi E, et al: Microsurgical posterior herniotomy with en bloc laminoplasty: alternative method for treating cervical disc herniation. J Spinal Disord Tech 18:171-177, 2005

62. Simpson JM, Silveri CP, Balderston RA, Simeone FA, An HS: The results of operations on the lumbar spine in patients who have diabetes mellitus. J Bone Joint Surg Am 75:18231829,1993

63. Song KJ, Lee KB, Song JH: Efficacy of multilevel anterior cervical discectomy and fusion versus corpectomy and fusion for multilevel cervical spondylotic myelopathy: a minimum 5-year follow-up study. Eur Spine J 21:1551-1557, 2012

64. Suk KS, Kim KT, Lee JH, Lee SH, Lim YJ, Kim JS: Sagittal alignment of the cervical spine after the laminoplasty. Spine (Phila Pa 1976) 32:E656-E660, 2007

65. Tracy JA, Bartleson JD: Cervical spondylotic myelopathy. Neurologist 16:176-187, 2010

66. Tsuji T, Asazuma T, Masuoka K, Yasuoka H, Motosuneya T, Sakai T, et al: Retrospective cohort study between selective and standard C3-7 laminoplasty. Minimum 2-year follow-up study. Eur Spine J 16:2072-2077, 2007

67. Uematsu Y, Tokuhashi Y, Matsuzaki H: Radiculopathy after laminoplasty of the cervical spine. Spine (Phila Pa 1976) 23:2057-2062, 1998

68. Wang HQ, Mak KC, Samartzis D, El-Fiky T, Wong YW, Luo ZJ, et al: "Spring-back" closure associated with open-door cervical laminoplasty. Spine J 11:832-838, 2011

69. Wilson JR, Fehlings MG, Kalsi-Ryan S, Shamji MF, Tetreault LA, Rhee JM, et al: Diagnosis, heritability, and outcome assessment in cervical myelopathy: a consensus statement. Spine (Phila Pa 1976) 38 (22 Suppl 1):S76-S77, 2013

70. Woods BI, Hohl J, Lee J, Donaldson W III, Kang J: Laminoplasty versus laminectomy and fusion for multilevel cervical spondylotic myelopathy. Clin Orthop Relat Res 469:688695, 2011

71. Xia Y, Xia Y, Shen Q, Li H, Xu T: Influence of hinge position on the effectiveness of expansive open-door laminoplasty for cervical spondylotic myelopathy. J Spinal Disord Tech 24:514-520, 2011

72. Yang L, Gu Y, Shi J, Gao R, Liu Y, Li J, et al: Modified plateonly open-door laminoplasty versus laminectomy and fusion for the treatment of cervical stenotic myelopathy. Orthopedics 36:e79-e87, 2013

73. Yonenobu K, Hosono N, Iwasaki M, Asano M, Ono K: Laminoplasty versus subtotal corpectomy. A comparative study of results in multisegmental cervical spondylotic myelopathy. Spine (Phila Pa 1976) 17:1281-1284, 1992

74. Yonenobu K, Hosono N, Iwasaki M, Asano M, Ono K: Neurologic complications of surgery for cervical compression myelopathy. Spine (Phila Pa 1976) 16:1277-1282, 1991

75. Yoshida G, Kanemura T, Ishikawa Y, Sakai Y, Iwase T, Matsuyama Y, Ishiguro N: Cardiopulmonary function in elderly patients with cervical spondylotic myelopathy. J Orthop Sci 17:3-8, 2012

76. Yoshida M, Tamaki T, Kawakami M, Nakatani N, Ando M, Yamada H, et al: Does reconstruction of posterior ligamentous complex with extensor musculature decrease axial symptoms after cervical laminoplasty? Spine (Phila Pa 1976) 27:1414-1418, 2002

\section{Disclosure}

The authors report no conflict of interest concerning the materials or methods used in this study or the findings specified in this paper.

\section{Author Contributions}

Conception and design: Fehlings, Tetreault, Ibrahim. Analysis and interpretation of data: Tetreault, Ibrahim, Côté, Singh. Drafting the article: all authors. Critically revising the article: all authors. Reviewed submitted version of manuscript: all authors. Approved the final version of the manuscript on behalf of all authors: Fehlings.

\section{Correspondence}

Michael G. Fehlings, The Toronto Western Hospital, University Health Network, Rm. 4W-449, 399 Bathurst St., Toronto, ON M5T 2S8, Canada. email: michael.fehlings@uhn.on.ca. 